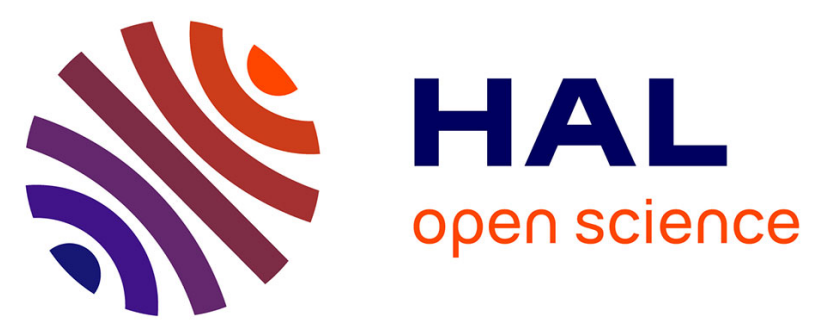

\title{
An explicit dynamics extended finite element method. Part 1: Mass lumping for arbitrary enrichment functions
} Thomas Elguedj, Anthony Gravouil, Hubert Maigre

\section{To cite this version:}

Thomas Elguedj, Anthony Gravouil, Hubert Maigre. An explicit dynamics extended finite element method. Part 1: Mass lumping for arbitrary enrichment functions. Computer Methods in Applied Mechanics and Engineering, 2009, 198, pp.2297-2317. 10.1016/j.cma.2009.02.019 . hal-00383288

\section{HAL Id: hal-00383288 \\ https://hal.science/hal-00383288}

Submitted on 12 May 2009

HAL is a multi-disciplinary open access archive for the deposit and dissemination of scientific research documents, whether they are published or not. The documents may come from teaching and research institutions in France or abroad, or from public or private research centers.
L'archive ouverte pluridisciplinaire HAL, est destinée au dépôt et à la diffusion de documents scientifiques de niveau recherche, publiés ou non, émanant des établissements d'enseignement et de recherche français ou étrangers, des laboratoires publics ou privés. 


\title{
An explicit dynamics extended finite element method. Part 1: Mass lumping for arbitrary enrichment functions.
}

\author{
T. Elguedj ${ }^{1}$, A. Gravouil , H. Maigre \\ Université de Lyon, CNRS \\ INSA-Lyon, LaMCoS, UMR5259 \\ 20 avenue Albert Einstein, F69621 Villeurbanne Cedex, France
}

\begin{abstract}
This paper presents a general mass lumping technique for explicit dynamics simulations using the eXtended Finite Element Method with arbitrary enrichment functions. The proposed mass lumping technique is a generalization of previously published results for cracks and holes. Time step estimates are studied for crack singular enrichment functions and for hole enrichment. In both cases, we show that the critical time step does not tend to zero and is of the same order as that of the same unenriched element. The performance of the method is demonstrated on several numerical examples that compare well with classical finite elements and previously published X-FEM results.
\end{abstract}

Key words: X-FEM; explicit dynamics; mass lumping; enrichment.

\section{Introduction}

The accurate prediction of the behavior of complex structures in fast transient applications is a difficult challenge from numerical and industrial points of view. Real-life applications such as crash, impact and explosions require very small time increments in order to resolve accurately the physical phenomenon involved. The computational cost of such numerical simulations with large meshes of complex non-linear structures can become problematic even though computer performance is growing rapidly. To overcome this, explicit time integrators (such as the central difference scheme, see, e.g., [29]) are very popular

$\overline{1}$ Corresponding author: thomas.elguedj@insa-lyon.fr 
in the solid mechanics community. They require small time steps for stability reasons, but these values are usually of the same order of the physical phenomenon that are modelled. Moreover, they do not require any iterations in non-linear cases and if used with appropriate mass lumping scheme, their computational cost can be reduced significantly as the diagonal matrix inversion is straightforward.

The modelling of discontinuities such as cracks and complex shapes in the finite element method requires conforming meshes that usually have a large dispersion in the mesh density. Because of the stability condition that limits the time step, these small elements impose a very small time step for the whole mesh. For the central difference scheme, superconvergence is observed when the time step equals the stability limit (see, e.g., [16]). This behavior leads us to compute such simulations with a time step as close to the critical time step as possible. However, a large dispersion in the mesh density forces us to "compute" most finite elements with a time step quite smaller than their stability limit. This is penalizing in term of computational cost, as it requires to compute more time steps, as well as for the quality of the solution.

Based on the Partition of Unity of Babuška and Melenk [1], the eXtended Finite Element Method was introduced by Belytschko and Black [4] and Moës et al. [26]. It was first applied to two-dimensional crack growth simulation by Belytschko and co-workers $[4,26]$, then to three-dimensional crack propagation in Sukumar et al. [38], Moës et al. [27] and Gravouil et al. [14]. It was further extended to many applications such as holes and inclusions in Sukumar et al. [37], Daux et al. [7] and more recently by Rozycki et al. [35]. The use of the X-FEM in dynamic situations was mostly focused on dynamic crack growth modelling. Belytschko et al. [3] were the first to point the problem of possibly null critical time step with X-FEM and therefore used the implicitexplicit element by element scheme of Hughes and Liu [17]. Réthoré et al. [33, 34] and Prabel et al. [30] circumvent this problem by using an implicit time integrator with a specific enrichment strategy to ensure energy conservation. De Borst et al. [8] and Remmers et al. [32] also experienced the critical time step problem with discontinuous enrichment. They proposed to prevent the crack from getting too close to the nodes and to choose very small time steps.

The only successful attempts in finding appropriate mass lumping schemes for enrichment that produce non-zero critical time steps are the ones of Menouillard et al. [24, 25] for dynamic crack growth and Rozycki et al. [35] for free boundaries and holes with constant strain elements. However, these two methods have certain drawbacks. The lumping scheme and critical time step estimates of Rozycki et al. is only valid for constant strain elements, that is triangular and tetrahedral finite elements. The various lumping techniques proposed by Menouillard et al. are designed for discontinuous enrichment only. The absence of a cohesive law on the crack faces or of singular en- 

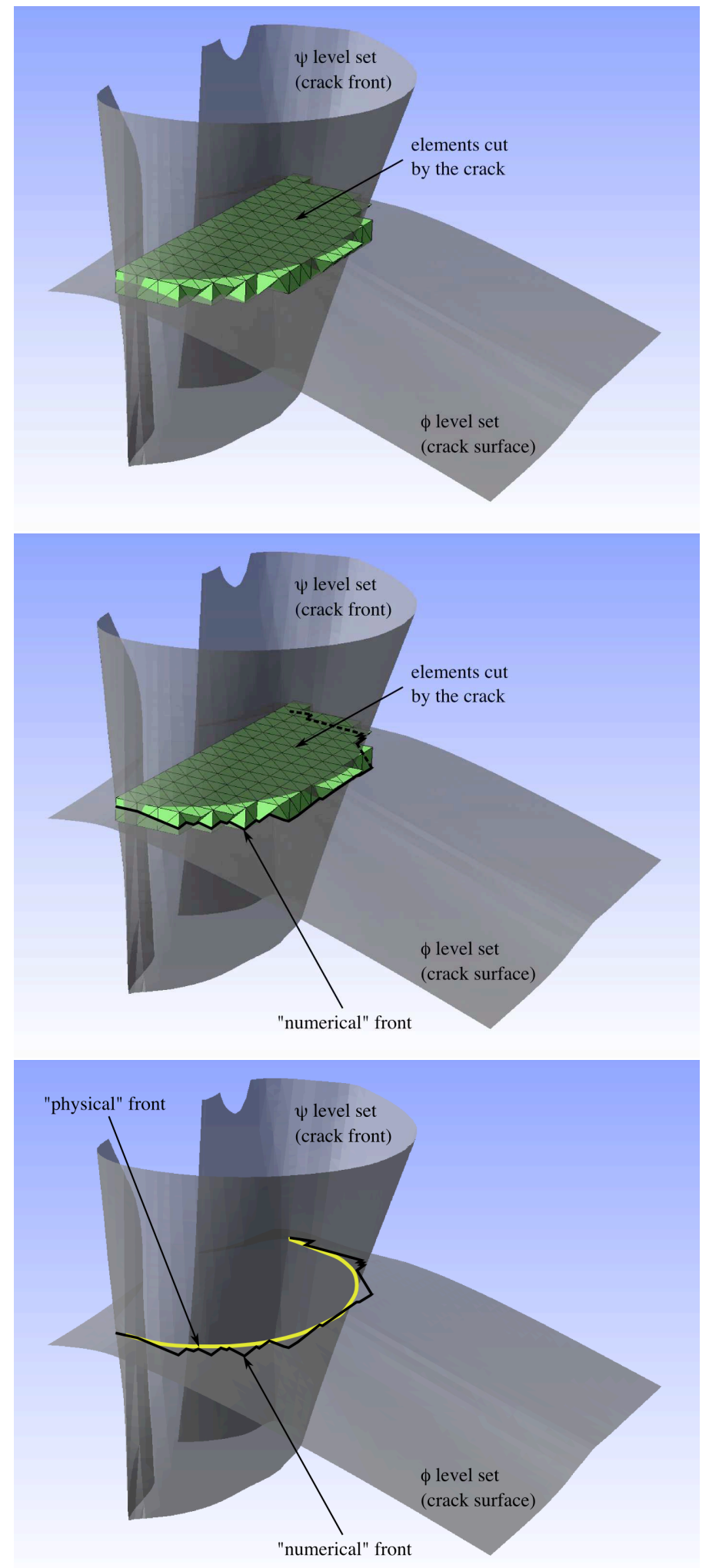

Fig. 1. Non-planar 3D crack: level set representation, elements cut by the crack, "numerical" and "physical" crack front with a pure discontinuous enrichment approach. 
richment prevents us to position accurately the crack front inside an element. The "numerical front" is always located on an element boundary, whereas the "physical front" can be located inside an element but is not modelled in the finite element approximation. In two-dimensional simulations, if the mesh is sufficiently fine, this has no visible effect on the crack path prediction as shown in Menouillard et al. citetomtom06,tomtom2. However, for three-dimensional simulations with non planar cracks, the faceted description of the front with a purely discontinuous X-FEM approach can become problematic as presented in Figure 1. This aspect is what motivates the present study. We want to model accurately three-dimensionnal fast dynamic phenomenons in complex structures, mainly crack propagation. We believe that from both computational and physical aspects, explicit time integrators are well suited for this purpose. Although all the examples that will be presented throughout the paper are two-dimensional ones, we want to develop a method that will be as efficient in three-dimensional cases. For the case of three-dimensional dynamic crack growth we believe that the use of singular enrichment is essential.

We propose in this paper a general mass lumping formula for arbitrary enrichment functions. It is based on an exact representation of the kinetic energy of rigid body modes and enrichment modes. We focus on the application of this expression to dynamics crack growth modelling with singular enrichment and of free boundaries and holes with quadrilateral meshes.

The paper is constructed as follows. In Section 2, the governing equations of explicit dynamics are recalled, a brief overview of the X-FEM is given with a particular emphasis on dynamic crack modelling. In Section 3, the general mass lumping formula is given and applied to cracks and holes modelling for which critical time steps estimates are presented and general rules are given. In Section 4, several numerical examples are presented where we compare the method to standard finite elements and other X-FEM approaches for dynamics crack growth simulations.

\section{Governing equations}

\subsection{Continuous formulation}

The strong form of the initial boundary value problem of structural dynamics for a body $\Omega$ can be stated as follows (see, e.g., Hughes [16]):

Given $\mathbf{f}: \Omega \times] 0 ; T\left[\rightarrow \mathbb{R}^{3}, \mathbf{g}: \Gamma_{g} \times\right] 0 ; T\left[\rightarrow \mathbb{R}^{3}\right.$, and $\left.\mathbf{h}: \Gamma_{h} \times\right] 0 ; T\left[\rightarrow \mathbb{R}^{3}\right.$, find 
$\mathbf{u}: \bar{\Omega} \rightarrow \mathbb{R}^{3}$ such that:

$$
\begin{aligned}
& \left.\rho \frac{\partial^{2} \mathbf{u}}{\partial t^{2}}=\operatorname{div} \boldsymbol{\sigma}+\mathbf{f} \quad \text { on } \Omega \times\right] 0 ; T[\text {, } \\
& \left.\mathbf{u}=\mathbf{g} \quad \text { on } \Gamma_{g} \times\right] 0 ; T[, \\
& \left.\boldsymbol{\sigma} \cdot \mathbf{n}=\mathbf{h} \quad \text { on } \Gamma_{h} \times\right] 0 ; T[, \\
& \mathbf{u}(\mathbf{x}, 0)=\mathbf{u}_{0}(\mathbf{x}) \quad \mathbf{x} \in \Omega, \\
& \frac{\partial \mathbf{u}}{\partial t}(\mathbf{x}, 0)=\dot{\mathbf{u}}_{0}(\mathbf{x}) \quad \mathbf{x} \in \Omega
\end{aligned}
$$

where $\mathbf{n}$ is the exterior unit normal on $\Gamma$, the boundary of $\Omega, \mathbf{g}$ is the prescribed displacement on $\Gamma_{g}$ and $\mathbf{h}$ is the prescribed traction on $\Gamma_{h}$, which form together the boundary $\Gamma=\Gamma_{h} \cup \Gamma_{g}$ of $\Omega$, and $\mathbf{f}$ is the body force. $\mathbf{u}_{0}$ and $\dot{\mathbf{u}}_{0}$ are the initial displacement and velocity conditions. The stress tensor $\boldsymbol{\sigma}$ is defined in terms of the strain tensor $\varepsilon$ by the generalized Hooke's law:

$$
\begin{aligned}
\boldsymbol{\varepsilon}=\nabla^{s} \mathbf{u} & =\frac{1}{2}\left(\nabla \mathbf{u}+\nabla \mathbf{u}^{T}\right), \\
\boldsymbol{\sigma} & =\mathbf{c}: \boldsymbol{\varepsilon} .
\end{aligned}
$$

Eq. (1) is the equation of motion, Eqs. (2) and (3) are the Dirichlet and Neumann boundary conditions and Eqs. (4) and (5) are the initial conditions.

We define the trial and weighting spaces as follows:

$$
\begin{aligned}
& \mathcal{S}_{t}=\left\{\mathbf{u}(\cdot, t) \mid \mathbf{u}(\mathbf{x}, t)=\mathbf{g}(\mathbf{x}, t), \mathbf{x} \in \Gamma_{g}, \mathbf{u}(\cdot, t) \in H^{1}(\Omega)\right\} \\
& \mathcal{V}=\left\{\mathbf{u}(\cdot, t) \mid \mathbf{u}(\mathbf{x}, t)=\mathbf{0}, \mathbf{x} \in \Gamma_{g}, \mathbf{u}(\cdot, t) \in H^{1}(\Omega)\right\}
\end{aligned}
$$

The weak form corresponding to Eqs. (1) to (5) can now be stated as:

Given $\mathbf{f}, \mathbf{g}, \mathbf{h}, \mathbf{u}_{0}$ and $\dot{\mathbf{u}}_{0}$, find $\mathbf{u}(t) \in \mathcal{S}_{t}, t \in[0 ; T]$, such that for all $\mathbf{w} \in \mathcal{V}$ :

$$
\begin{aligned}
(\mathbf{w}, \rho \ddot{\mathbf{u}})+a(\mathbf{w}, \mathbf{u}) & =(\mathbf{w}, \mathbf{f})+(\mathbf{w}, \mathbf{h})_{\Gamma_{h}} \\
(\mathbf{w}, \rho \mathbf{u}(0)) & =\left(\mathbf{w}, \rho \mathbf{u}_{0}\right) \\
(\mathbf{w}, \rho \dot{\mathbf{u}}(0)) & =\left(\mathbf{w}, \rho \dot{\mathbf{u}}_{0}\right)
\end{aligned}
$$

where standard notation for the $L^{2}$ inner product has been used:

$$
\begin{aligned}
(\mathbf{w}, \rho \ddot{\mathbf{u}}) & =\int_{\Omega} \mathbf{w} \cdot \rho \ddot{\mathbf{u}} d \Omega \\
a(\mathbf{w}, \mathbf{u}) & =\int_{\Omega} \varepsilon_{i j}(\mathbf{w}) c_{i j k l} \varepsilon_{k l}(\mathbf{u}) d \Omega \\
(\mathbf{w}, \mathbf{h})_{\Gamma_{h}} & =\int_{\Gamma_{h}} \mathbf{w} \cdot \mathbf{h} d \Gamma
\end{aligned}
$$




\subsection{Discrete formulation}

The semi-discrete Galerkin formulation of elastodynamics is:

Given $\mathbf{f}, \mathbf{g}, \mathbf{h}, \mathbf{u}_{0}$ and $\dot{\mathbf{u}}_{0}$, find $\mathbf{u}^{h}=\mathbf{v}^{h}+\mathbf{g}^{h}, \mathbf{u}^{h}(t) \in \mathcal{S}_{t}^{h}$ such that for all $\mathbf{w}^{h} \in \mathcal{V}^{h}:$

$$
\begin{aligned}
\left(\mathbf{w}^{h}, \rho \ddot{\mathbf{v}}^{h}\right)+a\left(\mathbf{w}^{h}, \mathbf{v}^{h}\right) & =\left(\mathbf{w}^{h}, \mathbf{f}\right)+\left(\mathbf{w}^{h}, \mathbf{h}\right)_{\Gamma_{h}}-\left(\mathbf{w}^{h}, \rho \ddot{\mathbf{g}}^{h}\right)-a\left(\mathbf{w}^{h}, \mathbf{g}^{h}\right), \\
\left(\mathbf{w}^{h}, \rho \mathbf{v}^{h}(0)\right) & =\left(\mathbf{w}^{h}, \rho \mathbf{u}_{0}\right)-\left(\mathbf{w}^{h}, \rho \mathbf{g}^{h}(0)\right) \\
\left(\mathbf{w}^{h}, \rho \dot{\mathbf{v}}^{h}(0)\right) & =\left(\mathbf{w}^{h}, \rho \dot{\mathbf{u}}_{0}\right)-\left(\mathbf{w}^{h}, \rho \dot{\mathbf{v}}^{h}(0)\right) .
\end{aligned}
$$

Using Eqs. (16) to (18) and the usual finite element interpolation for $\mathbf{u}^{h}$ and $\mathbf{w}^{h}$, we obtain the classical matrix problem:

$$
\begin{aligned}
M \ddot{U}+K U & =F \quad t \in] 0 ; T[, \\
U(0) & =U_{0}, \\
\dot{U}(0) & =\dot{U}_{0},
\end{aligned}
$$

where $M$ and $K$ are the usual mass and stiffness matrices, $F$ is the vector of applied forces and $\ddot{U}, \dot{U}, U$ are the acceleration, velocity and displacement vectors.

\subsubsection{Time discretization: explicit dynamics central difference scheme}

The previous equations constitute a coupled system of second order ordinary differential equations that needs to be integrated in time. This is done using Newmark's scheme:

$$
\begin{aligned}
& U_{n+1}=U_{n}+\Delta t \dot{U}_{n}+\Delta t^{2}\left(\frac{1}{2}-\beta\right) \ddot{U}_{n}+\Delta t^{2} \beta \ddot{U}_{n+1}, \\
& \dot{U}_{n+1}=\dot{U}_{n}+\Delta t(1-\gamma) \ddot{U}_{n}+\Delta t \gamma \ddot{U}_{n+1},
\end{aligned}
$$

where $\gamma$ and $\beta$ are the two parameters of the scheme. The stability and the order of accuracy of the scheme depend on the respective values of these two parameters.

The stability of the scheme can be studied using the energy method (see Hughes [16], chapter 9). It can be recast in the following form:

$$
\begin{cases}\frac{1}{2} \leq \gamma \leq \beta & \text { unconditionally stable } \\ \frac{1}{2} \leq \gamma \text { and } 2 \beta \leq \gamma & \text { stable if } \Delta t \leq \frac{1}{\omega_{\max } \sqrt{\gamma / 2-\beta}}\end{cases}
$$

where $\omega_{\max }$ is the largest eigenpulsation of the system. The scheme is secondorder accurate if $\gamma=\frac{1}{2}$. 
From now on we will use the explicit central difference scheme, which corresponds to Newmark's scheme with $\gamma=\frac{1}{2}$ and $\beta=0$. This scheme is explicit, second-order accurate and conditionally stable. The equations discretized in space and time are:

$$
\begin{aligned}
U_{n+1} & =U_{n}+\Delta t \dot{U}_{n}+\frac{\Delta t^{2}}{2} \ddot{U}_{n}, \\
M \ddot{U}_{n+1} & =F_{n+1}-F_{n+1}^{i n t}, \\
\dot{U}_{n+1} & =\dot{U}_{n}+\frac{\Delta t}{2}\left(\ddot{U}_{n}+\ddot{U}_{n+1}\right) .
\end{aligned}
$$

For a linear elastic material, we have $F_{n+1}^{i n t}=K U_{n+1}$, for a non-linear material, the internal forces are integrated from the stresses. The scheme being conditionally stable, the time step must be chosen in accordance with the Courant-Freidrich-Lewy condition:

$$
\Delta t \leq \Delta t_{c}=\frac{2}{\omega_{\max }}
$$

It can be seen from Eq. (25) that the update of the displacement only requires variables known from the previous time step. Consequently, solving the balance of momentum given by Eq. (26) does not require any iterations once the internal forces are assembled when considering a non-linear material. Finally if the mass matrix is lumped, that is replaced by a diagonal form, solving Eq. (26) is straightforward.

\subsubsection{The eXtended Finite Element Method}

The X-FEM was first introduced in Belytschko and Black [4] and Moës et al. [26]. Based on a local partition of unity (see Babuška and Melenk [1]), it consists in a finite element method where enrichment functions are added to the standard approximation. If we consider a set of $n_{e n r}$ scalar enrichment functions $\psi_{\alpha}(\mathbf{x})$, the displacement field can be written:

$$
u_{i}(\mathbf{x})=\sum_{A \in \mathcal{N}} u_{i}^{A} N^{A}(\mathbf{x})+\sum_{B \in \mathcal{N}_{\text {enr }}} \sum_{\alpha=1}^{n_{e n r}} b_{\alpha i}^{B} N^{B}(\mathbf{x}) \psi_{\alpha}(\mathbf{x})
$$

where $\mathcal{N}$ is the set of all the nodes in the mesh considered, $N^{A}(\mathbf{x})$ is the standard finite element shape function associated with node $A, u_{i}^{A}$ is the $i^{\text {th }}$ component of the associated degree of freedom, $\mathcal{N}_{e n r}$ is the set of enriched nodes and $b_{\alpha i}^{B}$ is the $i^{\text {th }}$ component of the enriched degree of freedom associated to node $B$ and to the $\alpha^{\text {th }}$ enriched function.

The X-FEM has already been used in various applications where appropriate 
enrichment functions were derived, based on asymptotic behaviour or smoothness considerations. The most frequent use of the method is to model discontinuities such as cracks, where two types of enrichments are utilized. The elements that are completely cut by a crack are enriched using a discontinuous function, such as the generalized Heaviside function or the Heaviside step function:

$$
H_{\text {gen }}(x)=\left\{\begin{array}{ll}
+1 & \text { if } x \geq 0, \\
-1 & \text { if } x<0 .
\end{array} \text { and } \quad H_{\text {step }}(x)=\left\{\begin{aligned}
+1 & \text { if } x \geq 0 \\
0 & \text { if } x<0
\end{aligned}\right.\right.
$$

The elements that contain a crack tip are enriched using functions that can represent the asymptotic displacement field. For linear elastic materials, these functions are derived from the Westergaard solutions (see, e.g., [4, 11]):

$$
\psi_{\alpha}(\mathbf{x})=\sqrt{r}\left[\sin \frac{\theta}{2}, \cos \frac{\theta}{2}, \sin \frac{\theta}{2} \sin \theta, \cos \frac{\theta}{2} \sin \theta\right],
$$

where $r$ and $\theta$ are polar coordinates centered at the crack tip.

For elastic-plastic materials with power-law hardening, enrichment functions derived from a Fourier analysis of the Hutchinson-Rice-Rosengren fields were proposed in Rao and Rahman [31] and Elguedj et al. [10]:

$$
\psi_{\alpha}(\mathbf{x})=r^{\frac{1}{n+1}}\left[\sin \frac{\theta}{2}, \cos \frac{\theta}{2}, \sin \frac{\theta}{2} \sin \theta, \cos \frac{\theta}{2} \sin \theta, \sin \frac{\theta}{2} \sin 3 \theta, \cos \frac{\theta}{2} \sin 3 \theta\right],
$$

where $n$ is the hardening exponent.

Another common use of the X-FEM is for free boundaries and holes as presented in Sukumar et al. [37], Daux et al. [7] and Rozycki et al. [35]. In this case, the displacement approximation for enriched elements contains only an enriched contribution, that is, Eq. (29) is replaced by:

$$
u_{i}(\mathbf{x})=\sum_{A \in \mathcal{N}_{e n r}} u_{i}^{A} N^{A}(\mathbf{x}) \psi(\mathbf{x})
$$

where the following enrichment function $V(x)$ is used:

$$
V(x)=\left\{\begin{aligned}
+1 & \text { if } x \text { belongs to the matter } \\
0 & \text { otherwise. }
\end{aligned}\right.
$$

An equivalent alternative in this case, which is preferred in numerical implementation, is to use a standard finite element interpolation and to perform the numerical integration only inside the matter. 


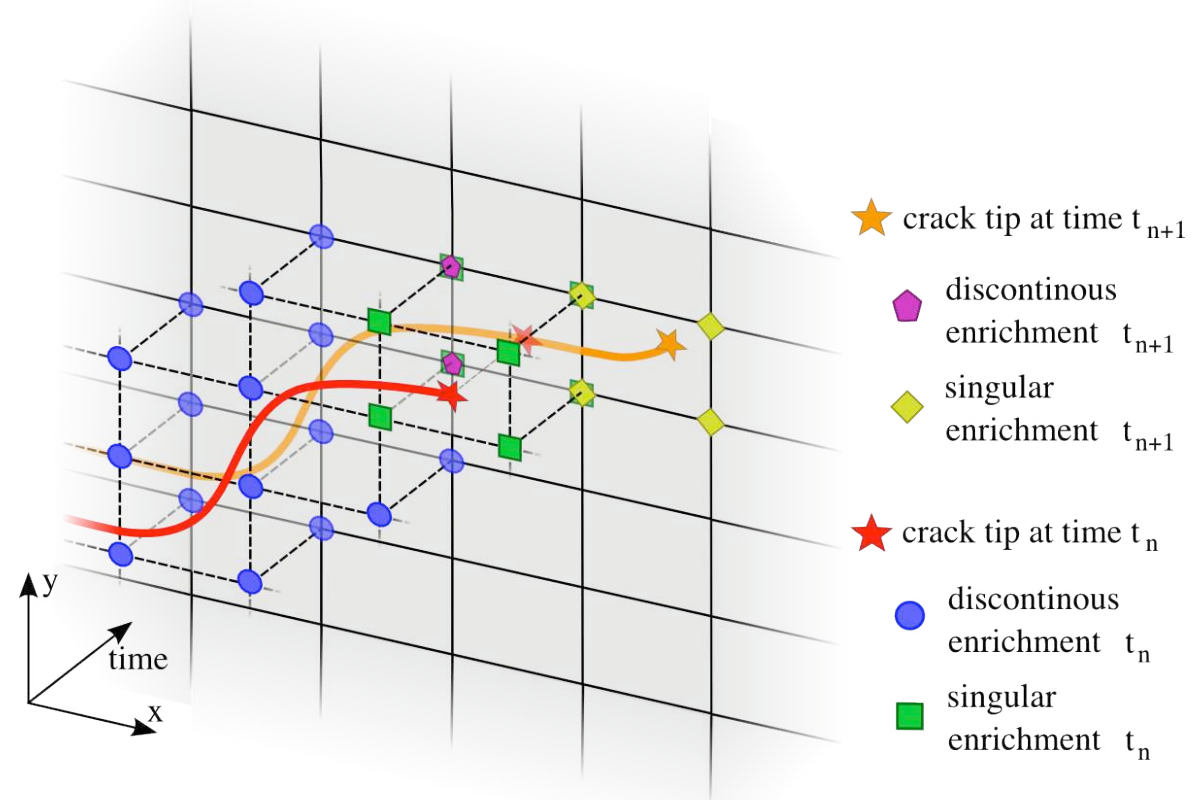

Fig. 2. Space-time representation of the crack, enriched elements and enriched nodes. "Old" enrichments $\left(t_{n}\right)$ are retained and new ones $\left(t_{n+1}\right)$ are created with respect to the new crack segment.

2.2.3.1 Numerical stability In the case of dynamic situations with moving discontinuities, such as dynamic crack growth, the question of numerical stability is important as enrichment functions evolve with time and so are the associated degrees of freedom. In our approach, we follow the same strategy as in Réthoré et al. [34] and Prabel et al. [30], that is we enrich all the mechanical fields (displacement, velocity, acceleration). The discrete problem can therefore be written as a classical finite element dynamic problem given in Eqs. (25) to (27).

Réthoré et al. [34] proved that to achieve energy conservation and numerical stability when the crack is growing, the following strategy should be adopted. Once the criteria for crack growth is met, all the "old" enrichments are retained and new ones, associated to the crack extension, are introduced. This idea is presented in Figure 2 with a space-time representation of the enrichments: when the crack grows from one element to an adjacent one, new singular enrichments are introduced on the nodes of the element containing the new tip. Discontinuous enrichments are added to nodes whose support becomes completely cut by the crack and do not have discontinuous enrichments yet. Considering a first order tensor $V$, we define the following notation, $V_{n}^{m}$ which denotes the value of $V$ at time $t_{n}$ expressed on the discretization (i.e. on the shape function basis) of time $t_{m}$. Energy conservation and numerical stability 
are achieved by using the balance recovery method (see Réthoré et al. [34]). By doing so, the kinetic and potential energies are conserved when the crack grows:

$$
U_{n}^{n+1 T} A_{n+1}^{n+1} U_{n}^{n+1}=U_{n}^{n T} A_{n}^{n} U_{n}^{n},
$$

where $A$ is either the mass matrix or the stiffness matrix. To obtain such a result, the values of the old enrichments are kept and the new ones initialized to zero. This corresponds to the following:

$$
\left[U_{n}^{n+1}\right]=\left[\begin{array}{c}
U_{n}^{n} \\
0 \\
\vdots \\
0
\end{array}\right] \quad \text { and } \quad\left[\dot{U}_{n}^{n+1}\right]=\left[\begin{array}{c}
\dot{U}_{n}^{n} \\
0 \\
\vdots \\
0
\end{array}\right] \quad \text { and } \quad\left[\ddot{U}_{n}^{n+1}\right]=\left[\begin{array}{c}
\ddot{U}_{n}^{n} \\
0 \\
\vdots \\
0
\end{array}\right]
$$

2.2.3.2 Fracture parameters and criteria It is relatively well admitted in the literature that brittle fracture is governed by the global macroscopic concept of Stress Intensity Factors introduced by Irwin [18], extended to elastodynamics within the framework of Bui [5] and Freund [12]. The dynamic Stress Intensity Factors in mode I and II, $K_{I}^{d y n}$ and $K_{I I}^{d y n}$, are given by:

$$
K_{I}^{d y n}=\lim _{r \rightarrow 0} \sqrt{2 \pi r} \sigma_{22}(\theta=0) \quad \text { and } \quad K_{I I}^{d y n}=\lim _{r \rightarrow 0} \sqrt{2 \pi r} \sigma_{12}(\theta=0) .
$$

We use the interaction integral approach (see, e.g., $[13,28]$ ) to compute the dynamic stress intensity factors as proposed by Krysl and Belytschko [21] and Réthoré et al. [34]. Once the dynamic stress intensity factors are computed, crack growth direction and velocity are determined using criteria and techniques that are relatively well established (see, for example, Maigre and Rittel [23]). Details on fracture parameters determination and criteria are given in Appendix A.

\section{Mass lumping and critical time steps for arbitrary enrichment functions}

\subsection{General expression based on kinetic energy conservation}

In order to obtain a general expression of the lumped mass for arbitrary enrichment functions, we follow the same idea as proposed in Menouillard et al. [24]. Let us consider a piecewise linear finite element in any dimension that is enriched with the arbitrary function $\psi$ at every node. The displacement 
at every point inside this element is given by Eq. (29). We seek an expression of the element lumped mass matrix that preserves the kinetic energy of the rigid body modes and the enrichment mode. For the standard degrees of freedom, we use classical mass lumping techniques such as the row-sum technique (see, e.g., [16]). For the enriched degrees of freedom, we consider the following. The enrichment function $\psi$ is imposed as Dirichlet velocity condition everywhere inside the element. The corresponding exact and discrete kinetic energies are:

$$
T=\frac{1}{2} \int_{\Omega_{e}} \rho \psi^{2} d \Omega_{e} \quad \text { and } \quad T^{h}=\frac{1}{2} \dot{U}_{x-f e m}^{T} M \dot{U}_{x-f e m} .
$$

If we replace the consistent mass matrix $M$ by a diagonal form $M_{L}$, the discrete kinetic energy becomes:

$$
T^{h}=\frac{1}{2} \sum_{i=1}^{\text {nnode }} m_{L i} \psi^{2}\left(\mathbf{x}_{i}\right),
$$

where $m_{L i}$ are the diagonal coefficients of $M_{L}, \mathbf{x}_{i}$ is the position of the $i^{\text {th }}$ node and nnode is the total number of nodes of the element considered.

Let us now suppose that the diagonal terms are identical $\left(m_{L i}=m_{L}, \forall i\right)$, that is all the enriched nodes have equal enriched masses. The conservation of the kinetic energy gives the following equation:

$$
\int_{\Omega_{e}} \rho \psi^{2} d \Omega_{e}=m_{L} \sum_{i=1}^{n n o d e} \psi^{2}\left(\mathbf{x}_{i}\right)
$$

from which we obtain the general expression of the diagonal enriched mass for an arbitrary enrichment function $\psi$ :

$$
m_{L}=\frac{1}{\sum_{i=1}^{n n o d e} \psi^{2}\left(\mathbf{x}_{i}\right)} \int_{\Omega_{e}} \rho \psi^{2} d \Omega_{e} .
$$

Remark 1 If we consider the generalized Heaviside function for $\psi$ as given in Eq. (30), the expression for the lumped mass coefficient becomes:

$$
\begin{aligned}
m_{L} & =\frac{1}{\sum_{i=1}^{\text {nnode }} 1^{2}} \int_{\Omega_{e}} \rho 1^{2} d \Omega_{e} \\
& =\frac{m_{\text {elt }}}{\text { nnode }} \frac{1}{\operatorname{mes}\left(\Omega_{e l}\right)} \int_{\Omega_{e}} \psi^{2} d \Omega_{e}
\end{aligned}
$$

which is the expression proposed by Menouillard et al. [24] for this particular case.

This shows that the general formula obtained in Eq. (41) agrees with previously published results for discontinuous enrichment functions and allows us 
to construct lumped mass matrix for any enrichment function. In the following, we concentrate our efforts on asymptotic crack enrichment functions and free boundaries enrichments.

In the following two paragraphs, we examine the proposed mass lumping technique on numerical examples. We compute numerically mass and stiffness matrices for one element with various enrichment functions. Then, we determine the maximum numerical eigenvalue for each case and therefore obtain critical time steps.

\subsection{Linear elastic fracture mechanics}

We first focus on the case of fracture mechanics, where the enrichment functions are given by Eq. (31). As presented in the introduction, these functions have two important roles: introduce more physics in the solution and localize the crack tip inside the element. In particular, the localization of the crack inside the element is done by the first enrichment function $\sqrt{r} \sin \frac{\theta}{2}$, as it is the only one which has a strong discontinuity inside the element (see Figure 3).

We propose to study the critical time step obtained by the lumping technique given in the previous paragraph as a function of the position of the crack tip inside an element. As shown in Figure 4, we study the case of a "stationary" crack (i.e. with only one tip inside the element) in a piecewise bilinear quadrilateral and a piecewise linear triangular element. We also study the case of a "moving" crack, that is we consider two successive crack tips inside the element with enriched functions associated to each tip. This case is a schematic representation of what happens when the crack grows but stays inside the same element. In this case, we use the enrichment strategy explained previously: each node contains several sets of asymptotic enrichment. Each set is associated with the successive crack tip positions within the element (two in the case considered).

In each case, we consider a parent quadrilateral or triangular element, the critical time step results are presented for lumped and consistent mass and are normalized by the critical time step obtained with a row-sum lumped mass for the same element without enrichment.

In Figures 5 and 6, the crack is cutting a piecewise bilinear quadrilateral element at $(-1 ; 0)$ on the center of the left edge of the element. In both figures, we plot the results with a unit scale and a zoomed scale. As for the case of the same element without enrichment, we can observe that the critical time step is lower with the consistent mass than with the lumped mass. With the consistent mass, the critical time step is maximum when the crack is almost completely cutting the element into two equal parts, whereas with the lumped 

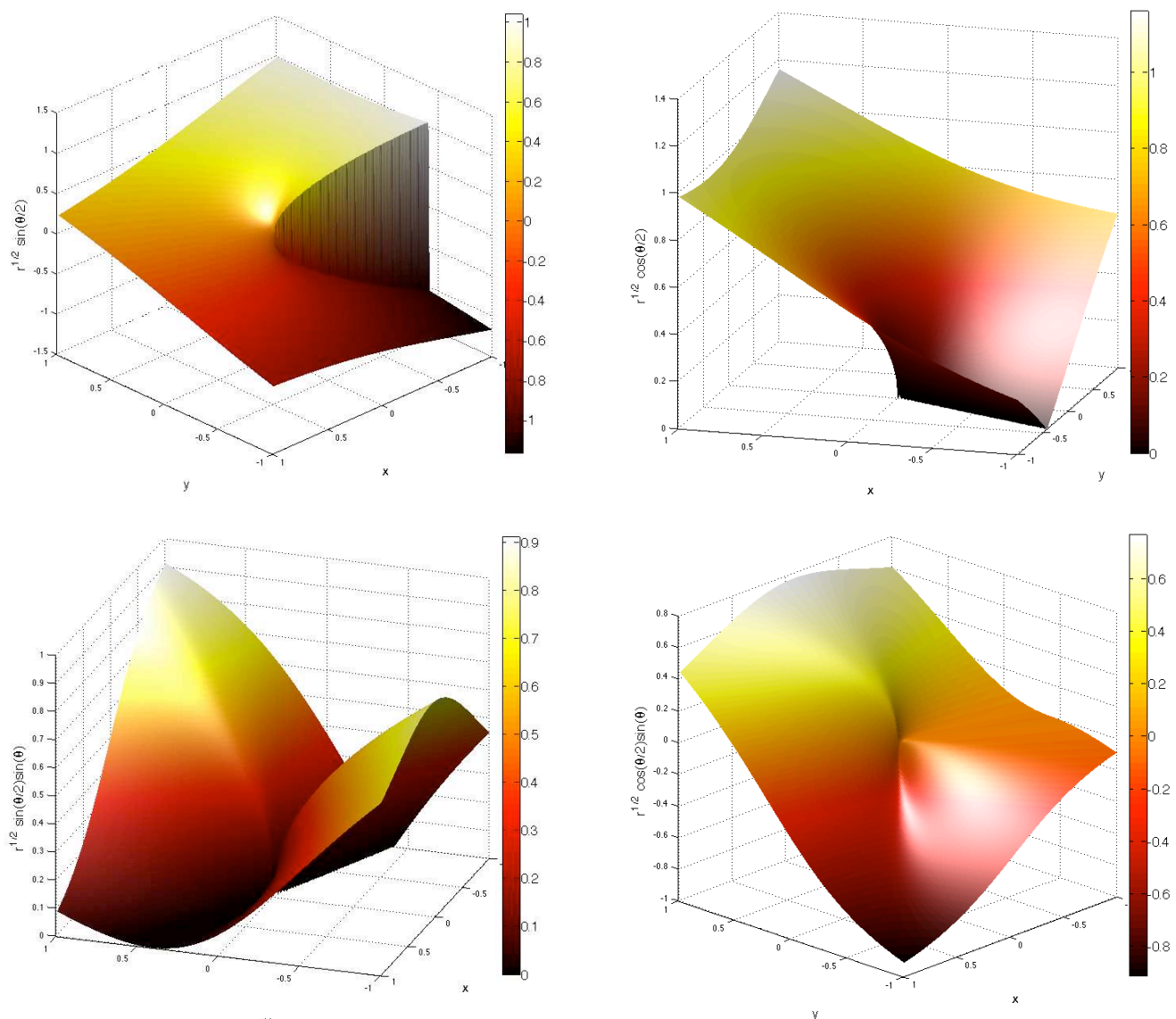

Fig. 3. $\sqrt{r} \sin \theta / 2$ (top left), $\sqrt{r} \cos \theta / 2$ (top right), $\sqrt{r} \sin \theta / 2 \sin \theta$ (bottom left), $\sqrt{r} \cos \theta / 2 \sin \theta$ (bottom right) enrichment functions on a parent element. The crack tip is located at the center of the element, the angle between the crack lips and the horizontal axis is $\pi / 8$.

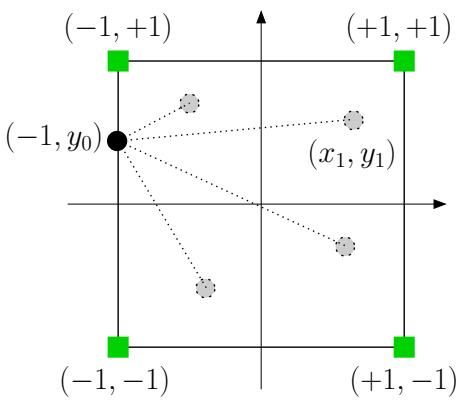

(a)

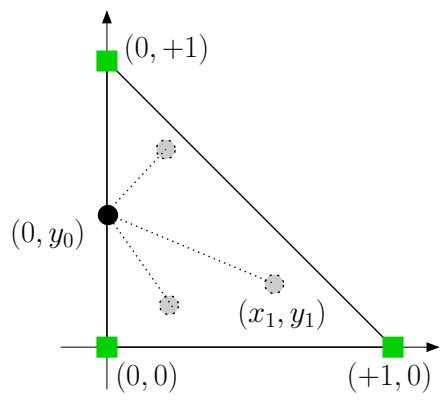

(b)

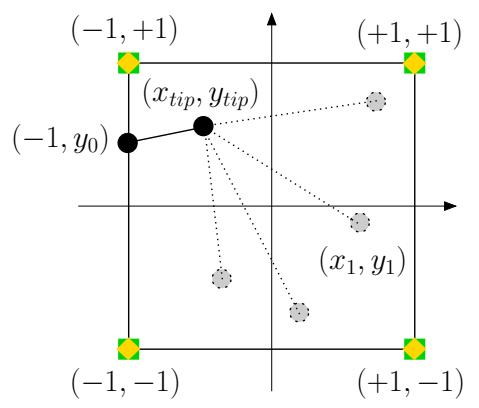

(c)

Fig. 4. Crack position in enriched parent elements used for critical time steps calculations. (a) Q4 element, (b) T3 element with stationary crack. (c) Q4 element with moving crack.

mass it is maximum when the tip is located around the center of the element.

In Figures 7 and 8, the crack is cutting a piecewise bilinear quadrilateral 

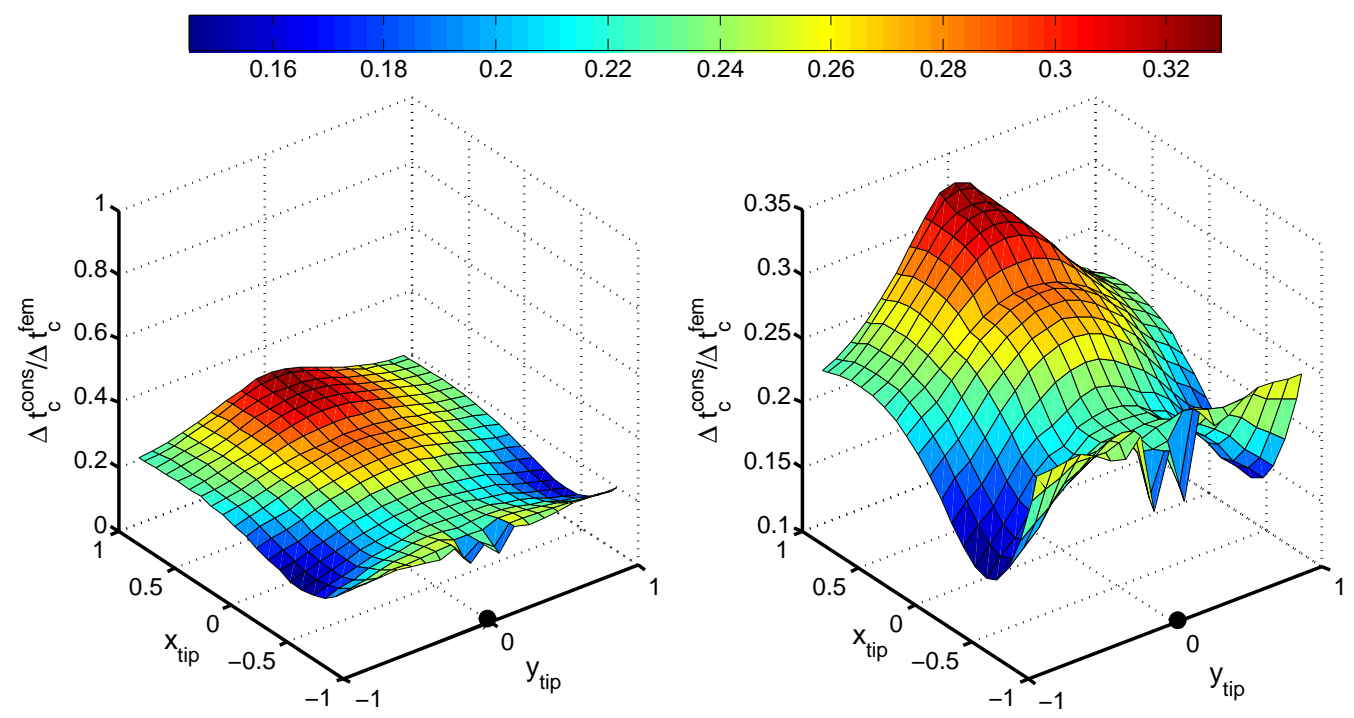

Fig. 5. Normalized critical time step (left, full $[0 ; 1]$ scale, right, zoomed scale) for the consistent mass matrix as a function of the crack tip position for a crack cutting an enriched Q4 element at $(-1 ; 0)$ (represented by the black dot).

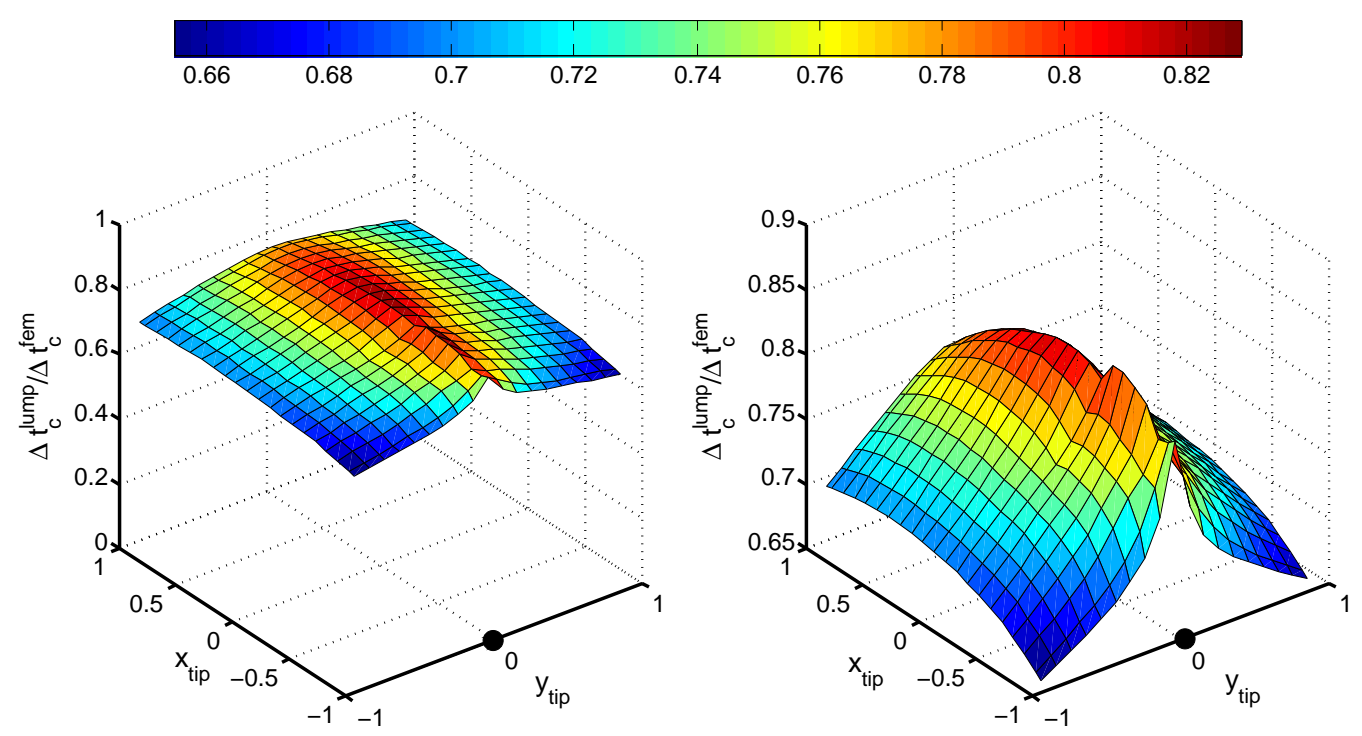

Fig. 6. Normalized critical time step (left, full $[0 ; 1]$ scale, right, zoomed scale) for the lumped mass matrix as a function of the crack tip position for a crack cutting an enriched Q4 element at $(-1 ; 0)$ (represented by the black dot).

element at $(-1 ; 0.75)$ around the top left corner of the element. In both figures, we plot the results with a unit scale and an zoomed scale. As for the previous case, the critical time step is lower with the consistent mass than with the lumped mass. With the consistent mass, the critical time step is maximum when the crack is almost cutting the element by a straight line in the direction of the bottom right corner. With the lumped mass, the critical time step is maximum when the crack tip is around the center of the element. 

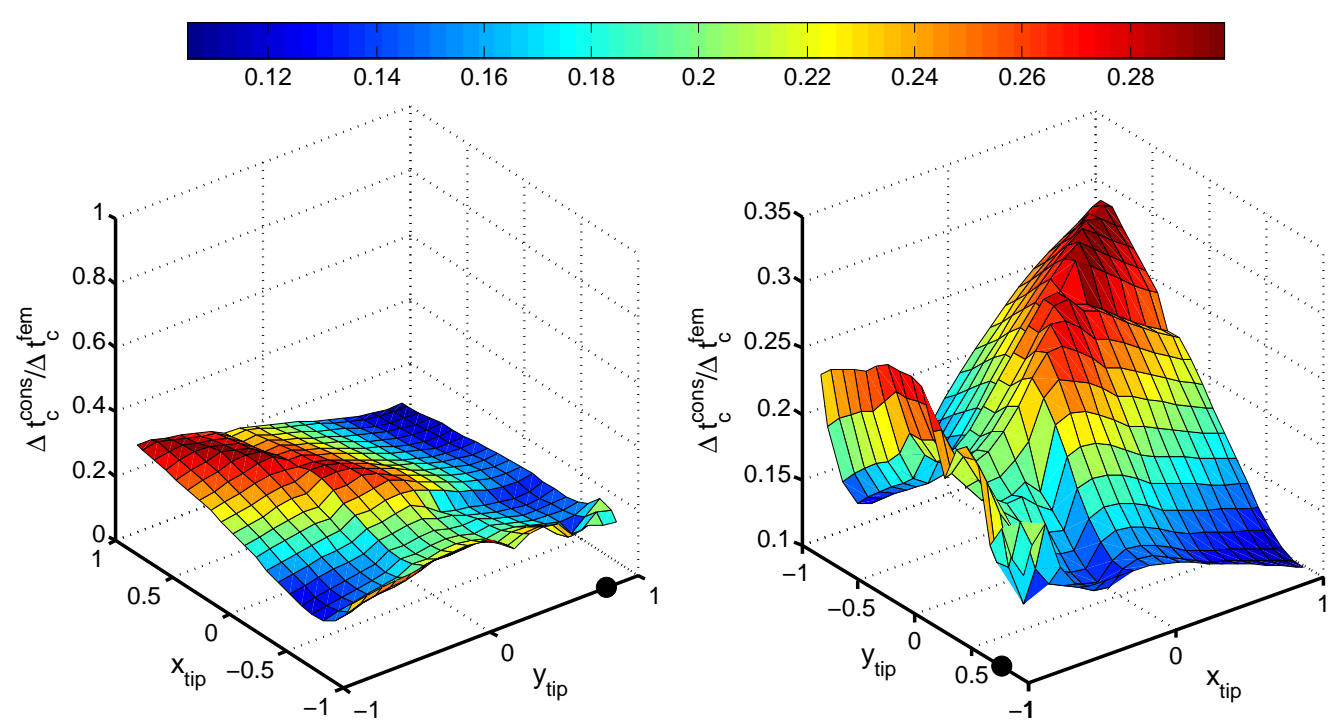

Fig. 7. Normalized critical time step (left, full $[0 ; 1]$ scale, right, zoomed scale) for the consistent mass matrix as a function of the crack tip position for a crack cutting an enriched Q4 element at $(-1 ; 0.75)$ (represented by the black dot).

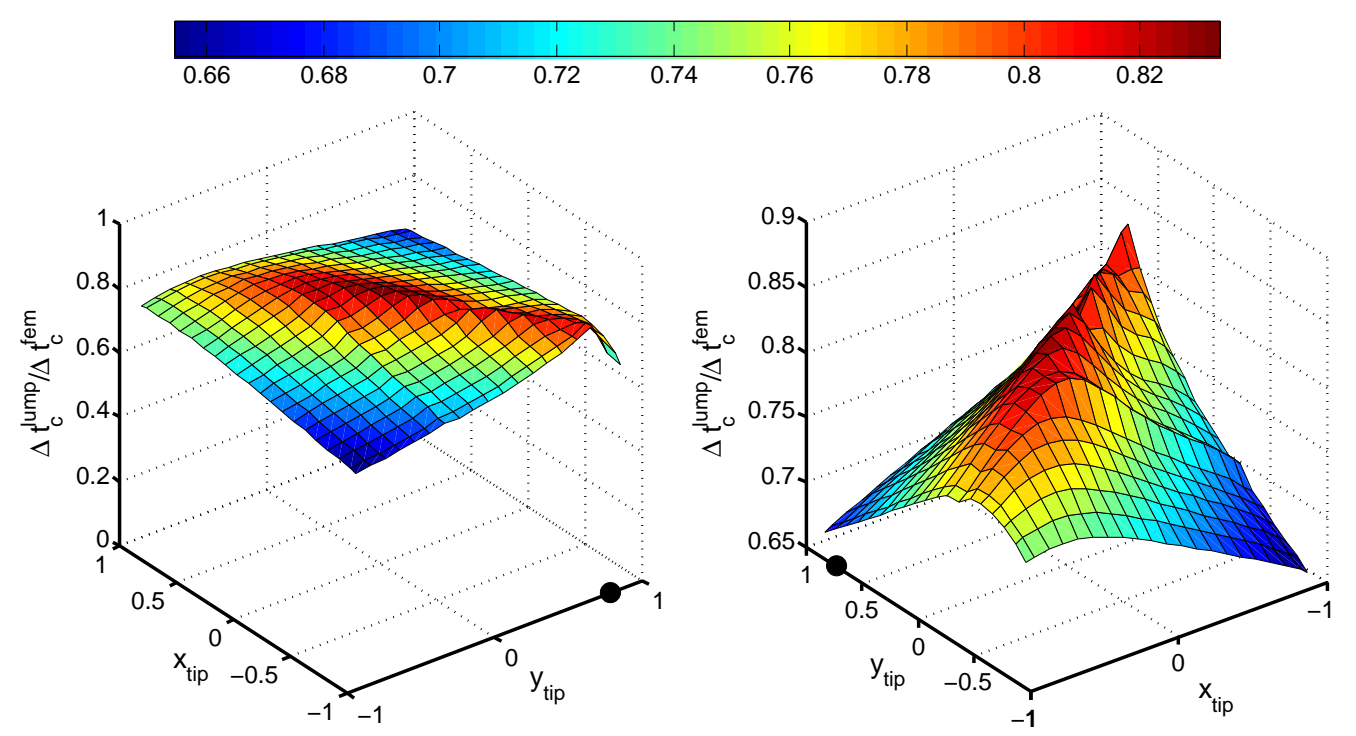

Fig. 8. Normalized critical time step (left, full $[0 ; 1]$ scale, right, zoomed scale) for the lumped mass matrix as a function of the crack tip position for a crack cutting an enriched Q4 element at $(-1 ; 0.75)$ (represented by the black dot).

In Figure 9, the crack is cutting a piecewise linear triangular element at $(0 ; 0.5)$ in the middle of the vertical edge of the element. We plot the normalized critical time step for the consistent mass on the left and for the lumped mass on the right. The zero values plotted are located outside the element. Again, the critical time step is lower with the consistent mass than with the lumped mass. In both cases, the value is maximum when the tip is located around the opposite node of the element. 

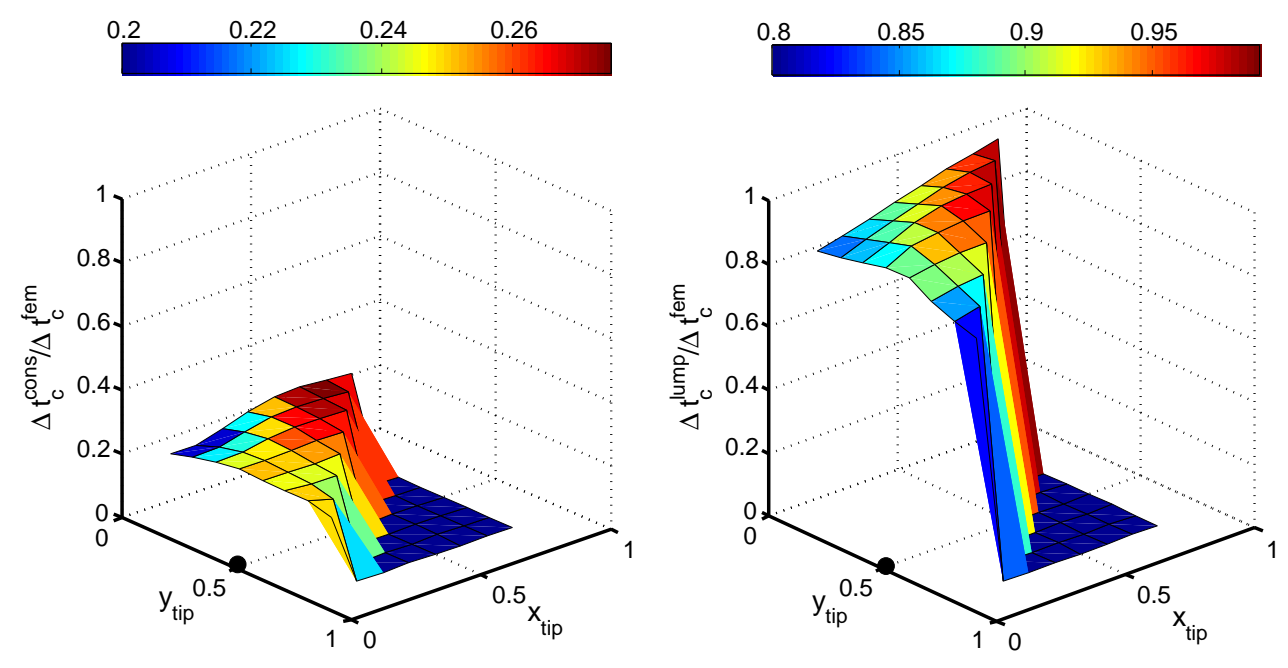

Fig. 9. Normalized critical time step for the consistent (left) and lumped (right) mass matrix as a function of the crack tip position for a crack cutting an enriched T3 element at $(0 ; 0.5)$ (represented by the black dot).
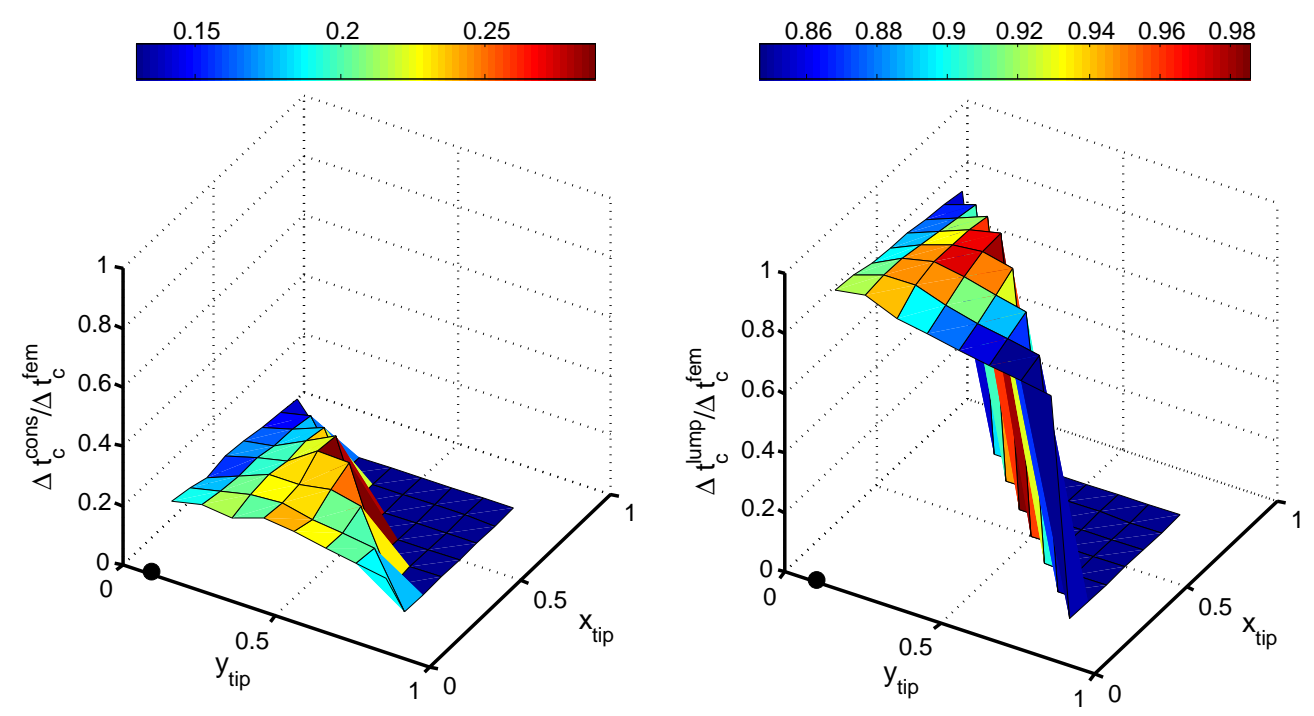

Fig. 10. Normalized critical time step for the consistent (left) and lumped (right) mass matrix as a function of the crack tip position for a crack cutting an enriched T3 element at $(0 ; 0.1)$ (represented by the black dot).

In Figure 10, the crack is cutting a piecewise linear triangular element at $(0 ; 0.1)$ around the bottom left node of the element. We plot the normalized critical time step for the consistent mass on the left and for the lumped mass on the right. The zero values plotted are located outside the element. Again, the critical time step is lower with the consistent mass than with the lumped mass. In both cases, the value is maximum when the tip is located around the center of the opposite edge of the element. 
In Figures 11 and 12, the crack is cutting a piecewise bilinear quadrilateral element at $(-1 ; 0.5)$ with initial crack tip located at $(-0.5 ; 0.5)$. We plot the critical time step as a function of the position of a moving tip location inside the same element, the angle between the two crack segments is limited to $-90^{\circ}$, that is the crack is not allowed to go backwards. With the consistent mass, the critical time step is maximum when the crack is almost completely cutting the element and the angle between the two crack segment is null. With the lumped mass, the critical time step is maximum when the angle between the two crack segments is around $-70^{\circ}$.
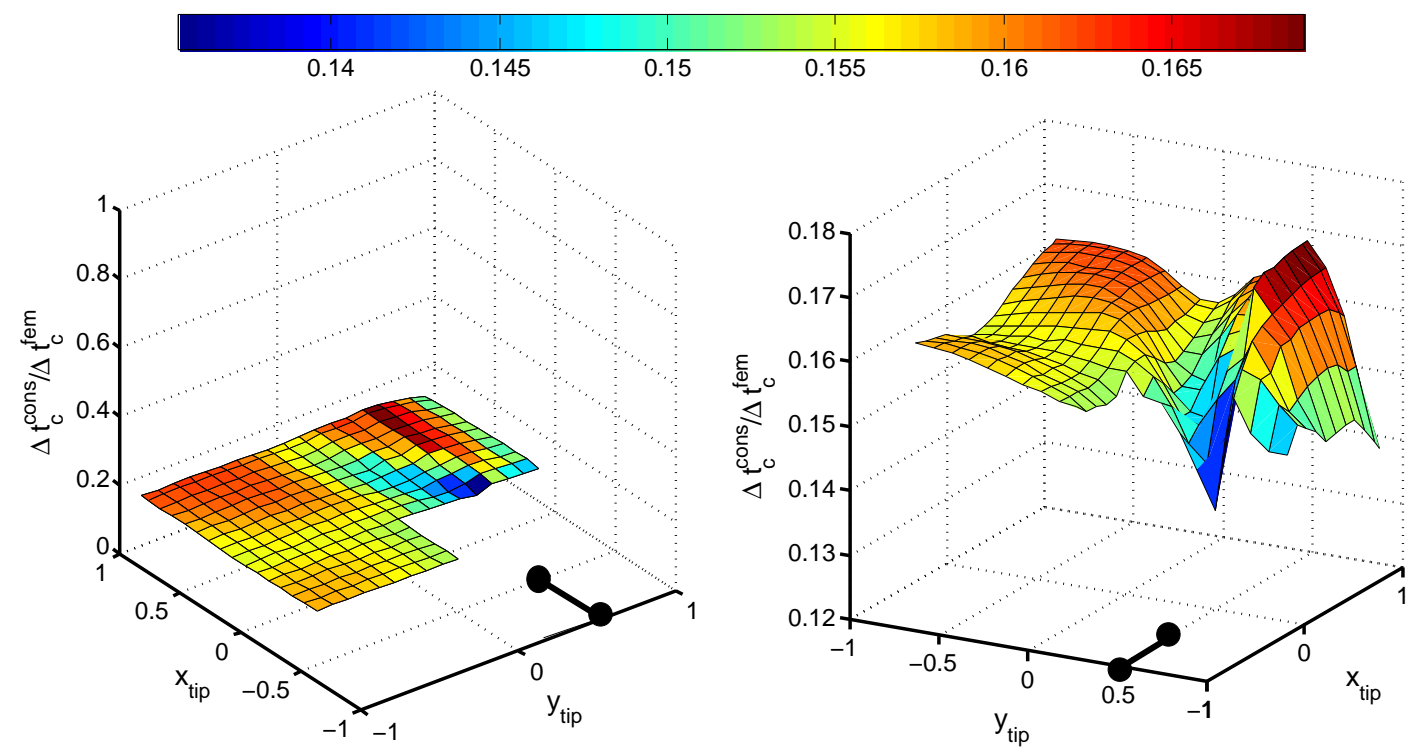

Fig. 11. Normalized critical time step (left, full $[0 ; 1]$ scale, right, zoomed scale) for the consistent mass matrix as a function of the propagating crack tip position for a crack cutting an enriched Q4 element at $(-1 ; 0.5)$ with initial tip at $(-0.5 ; 0.5)$ (represented by the black dots).

All the results for the minimum and maximum values for the critical time steps considering consistent and lumped mass matrices are given in Table 1. The conclusion of this study is that with the proposed mass lumping formula for the near tip function $\sqrt{r} \sin \frac{\theta}{2}$, the critical time step for a stationary crack is greater or equal than two-thirds of the critical time step of the same unenriched element with lumped mass, $\Delta t_{c \text { stat }}^{x-f e m} \geq \frac{2}{3} \Delta t_{c}^{f e m}$. This is a generalization to near tip singular enrichment of the formula proposed for discontinuous enrichment in Menouillard et al. [24]. With a crack moving inside the same element, the critical time step is greater or equal than one half of the critical time step of the same unenriched element with lumped mass, $\Delta t_{c \text { mov }}^{x-f e m} \geq \frac{1}{2} \Delta t_{c}^{f e m}$. Consequently, we propose to adopt for X-FEM explicit dynamics crack growth simulation with discontinuous and near tip asymptotic enrichments, the lumped mass formula given in Eq. (41) and the following expression for the critical time step:

$$
\Delta t_{c}^{x-f e m}=\frac{\Delta t_{c}^{f e m}}{2}
$$



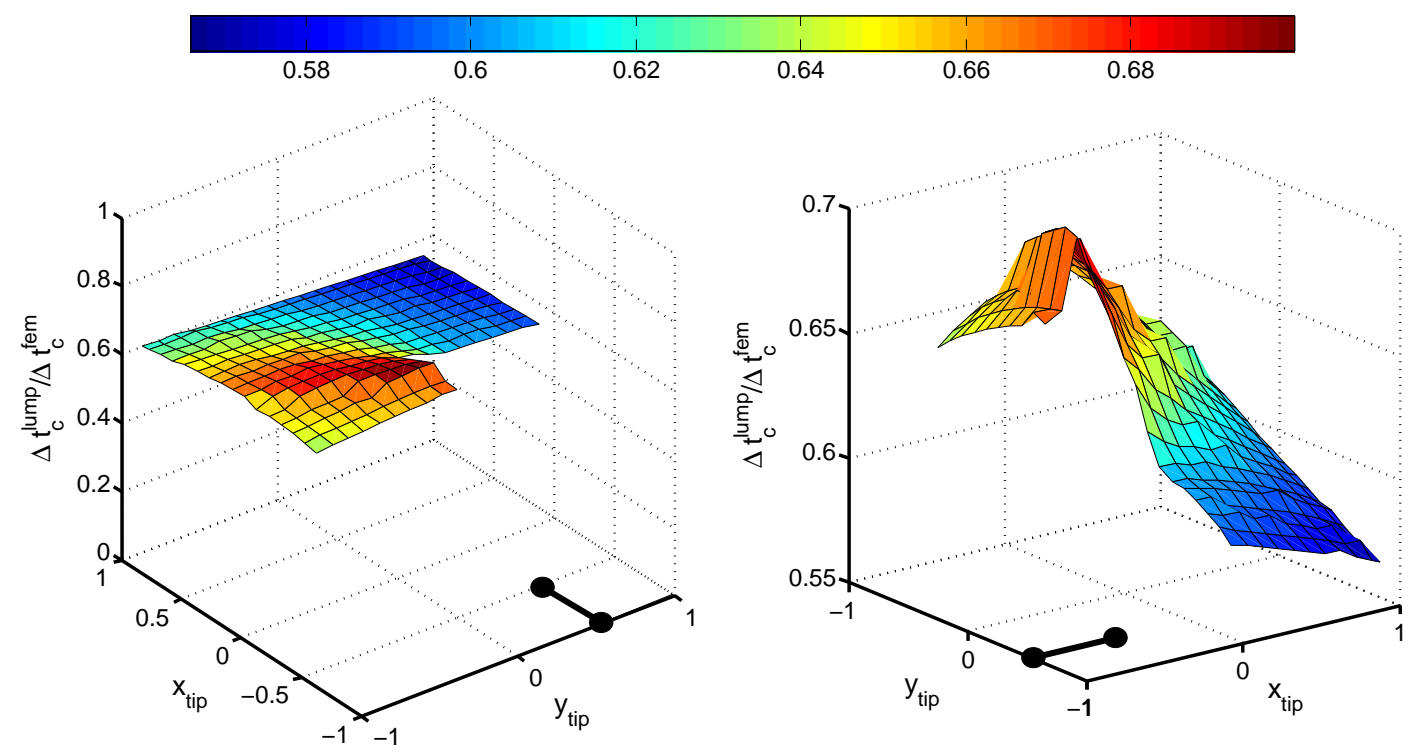

Fig. 12. Normalized critical time step (left, full $[0 ; 1]$ scale, right, zoomed scale) for the lumped mass matrix as a function of the propagating crack tip position for a crack cutting an enriched Q4 element at $(-1 ; 0.5)$ with initial tip at $(-0.5 ; 0.5)$ (represented by the black dots).

Table 1

\begin{tabular}{|c|c|c|c|c|}
\hline & \multicolumn{2}{|c|}{$\begin{array}{c}\text { Min value for } \\
\Delta t_{c}^{x-f e m} / \Delta t_{c}^{f e m}\end{array}$} & \multicolumn{2}{c|}{$\begin{array}{c}\text { Max value for } \\
\Delta t_{c}^{x-f e m} / \Delta t_{c}^{f e m}\end{array}$} \\
\cline { 2 - 5 } & lumped & consistent & lumped & consistent \\
\hline Q4 cut at $(-1 ; 0)$ & 0.66 & 0.15 & 0.82 & 0.32 \\
\hline Q4 cut at $(-1 ; 0.75)$ & 0.66 & 0.12 & 0.82 & 0.28 \\
\hline T3 cut at $(0 ; 0.5)$ & 0.8 & 0.2 & 0.95 & 0.26 \\
\hline T3 cut at $(0 ; 0.1)$ & 0.86 & 0.15 & 0.98 & 0.25 \\
\hline Q4 moving crack & 0.58 & 0.14 & 0.68 & 0.165 \\
\hline
\end{tabular}

Minimum and maximum values for the normalized critical time steps for asymptotic crack enrichment.

Remark 2 Although we only present results for a few configurations, we have tested various other ones and have always obtained similar results. The expression we propose for the definition of the X-FEM critical time step is indeed empirical, based on these numerical calculations. However, we believe that with reasonable care from a numerical and physical point of view in the simulation (for example crack increments comparable to the mesh size, ....) it will be verified as we will see in the examples. 


\subsection{Free boundaries and holes}

The case of holes and free boundaries has already been studied with explicit time integration schemes for constant strain elements, that is piecewise linear triangles and tetrahedrons, by Rozycki et al. [35]. The main difference in this case is that the displacement field contains only an enriched contribution as presented in Eq. (34). Thus, the previous analysis does not apply: the enriched mode is included in the rigid body modes. We impose a unit function $\bar{V}$ as a Dirichlet velocity condition everywhere inside the element in the horizontal or vertical direction. The corresponding exact and discrete kinetic energies are:

$$
T=\frac{1}{2} \int_{\Omega_{e} \backslash \Omega_{v o i d}} \rho \bar{V}^{2} d \Omega_{e} \quad \text { and } \quad T^{h}=\frac{1}{2} \dot{U}_{x-f e m}^{T} M \dot{U}_{x-f e m},
$$

where $\Omega_{\text {void }}$ is the part of $\Omega_{e}$ that does not belong to the matter. If we replace $M$ by a diagonal form $M_{L}$, the discrete kinetic energy becomes:

$$
T^{h}=\frac{1}{2} \sum_{i=1}^{\text {nnode }} m_{L i} \bar{V}^{2}\left(\mathbf{x}_{i}\right) .
$$

Again, we suppose that the diagonal terms are identical $\left(m_{L i}=m_{L}, \forall i\right)$, that is all the nodes have equal masses. The conservation of the kinetic energy gives the following equation:

$$
\int_{\Omega_{e}} \rho \bar{V}^{2} d \Omega_{e}=m_{L} \sum_{i=1}^{n \text { nnode }} \bar{V}^{2}\left(\mathbf{x}_{i}\right),
$$

from which we obtain the general expression of the diagonal enriched mass for this particular case:

$$
m_{L}=\epsilon \frac{m_{\text {elt }}}{\text { nnode }}
$$

where $\epsilon=\frac{\int_{\Omega_{e} \backslash \Omega_{v o i d}} d \Omega}{\int_{\Omega_{e}} d \Omega}$ is the material fraction of the element and $m_{\text {elt }}$ is the total mass of the element. It can be noted from Eq. (47) that the lumped mass matrix for any piecewise linear finite element cut by a hole can be obtained by multiplying the material fraction of the element by the lumped mass of the same element without enrichment (calculated from a row-sum technique):

$$
M_{L}^{x-f e m}=\epsilon M_{L}^{f e m} .
$$

This expression for the lumped mass is a generalization of the one proposed by Rozycki et al. [35] for piecewise linear triangles and tetrahedrons to piecewise bilinear quadrilaterals and trilinear hexahedrons.

It is important to note here, as presented in Rozycki et al. [35], that the stiffness matrix of a cut constant strain element can easily be obtained from 
the one of the same uncut element, as for the proposed lumped mass:

$$
K_{T 3}^{x-f e m}=\epsilon K_{T 3}^{f e m} \quad \text { and } \quad K_{T 6}^{x-f e m}=\epsilon K_{T 6}^{f e m} .
$$

Thus the critical time step for these cut elements is identical to one of the same uncut element.

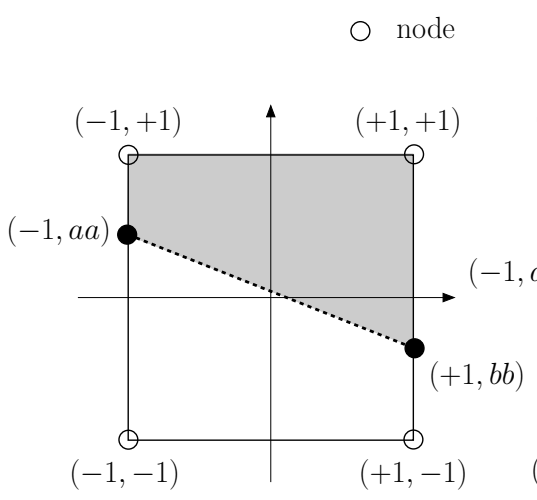

(a)
- -...... matter limit

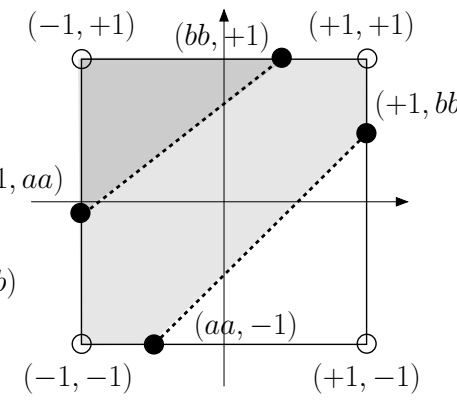

(b)

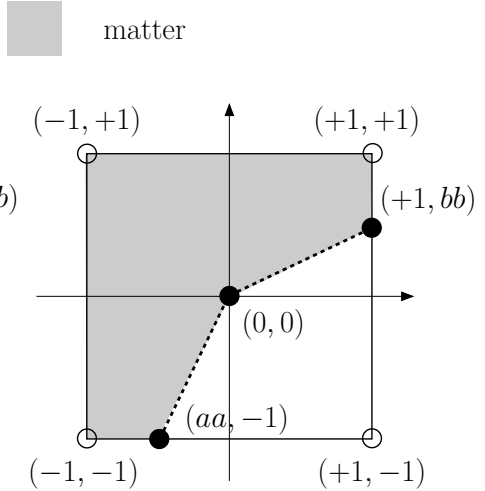

(c)

Fig. 13. Matter limit position in Q4 enriched parent elements used for critical time steps calculations. (a) Element cut by one segment, opposite edges. (b) Element cut by one segment, adjacent edges. (c) Element cut by two segments.
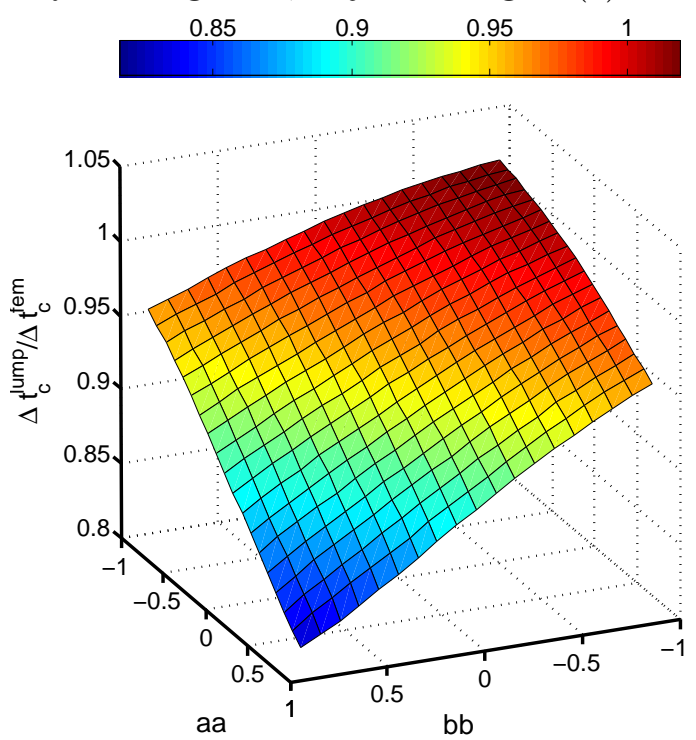
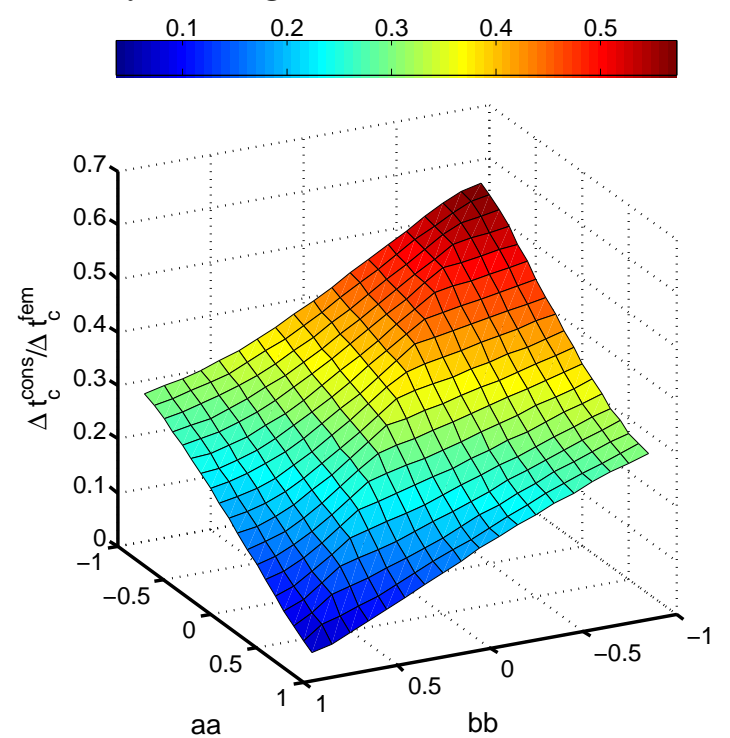

Fig. 14. Normalized critical time step for the lumped (left) and consistent (right) mass matrix as a function of the matter limit position: element cut by one segment, opposite left and right edges.

For piecewise bilinear quadrilaterals and piecewise trilinear hexahedrons, Eq. (49) is not verified. It is necessary to study the critical time step for these elements in order to obtain a rule similar to the one proposed in the previous paragraph for singular enrichment. We study the case of a piecewise bilinear quadrilateral cut by one segment from opposite and adjacent edges, and the case of the same element cut by two segment, as presented in Figure 13. The critical time 
step is obtained with the consistent mass matrix and the lumped mass matrix and is normalized by the critical time step of the same uncut element.

In Figure 14, the element is cut from opposite edges. The initial and final points position vary from the top right corner to the bottom right corner and from the top left corner to the bottom left corner. The critical time step is lower with the consistent mass than with the lumped mass. In both cases, the critical time step is minimum when the element is almost completely cut $(a a \simeq+1, b b \simeq+1)$ and maximum when the element is almost uncut $(a a \simeq-1, b b \simeq-1)$.
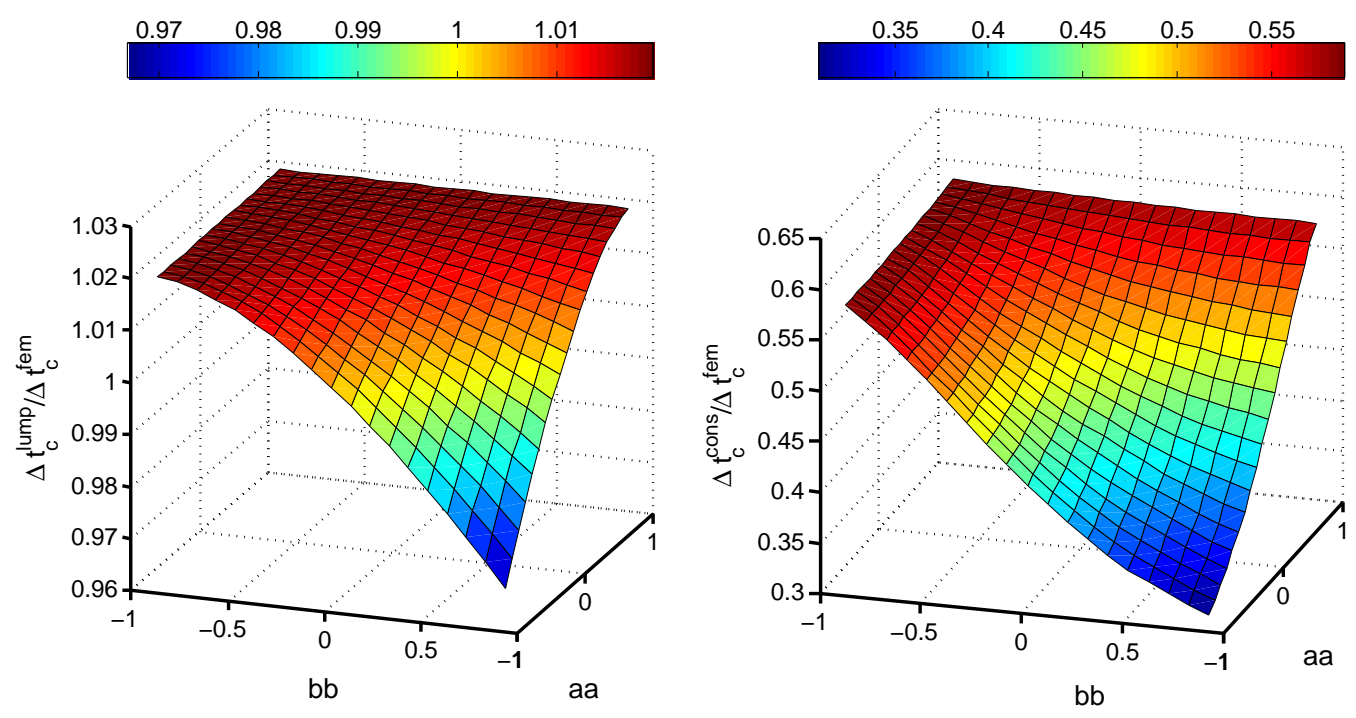

Fig. 15. Normalized critical time step for the lumped (left) and consistent (right) mass matrix as a function of the matter limit position: element cut by one segment, adjacent bottom and right edges.

In Figure 15, the element is cut from adjacent bottom and right edges. The initial and final points position vary from the top right corner to the bottom right corner and from the bottom left corner to the bottom right corner. The critical time step is again lower with the consistent mass than with the lumped mass. In both cases, it is minimum when the element is almost cut into two identical triangles $(a a \simeq-1, b b \simeq+1)$ and it is maximum when the element is almost uncut $(a a \simeq+1, b b \simeq+1, a a \simeq-1, b b \simeq-1$ and $a a \simeq+1, b b \simeq-1)$. In Figure 16, the element is cut from adjacent left and top edges. The initial and final points position vary from the bottom left corner to the top left corner and from the top right corner to the top left corner. The critical time step is again lower with the consistent mass than with the lumped mass. In both cases, it is minimum when the element is almost completely cut $(a a \simeq+1, b b \simeq-1)$ and it is maximum when the element is almost cut into two identical triangles $(a a \simeq-1, b b \simeq+1)$. 


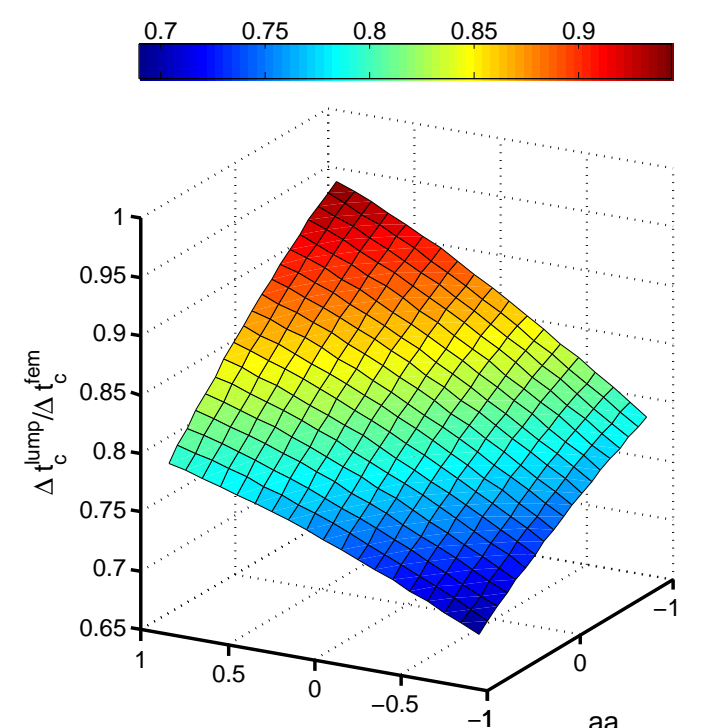

$\mathrm{bb}$

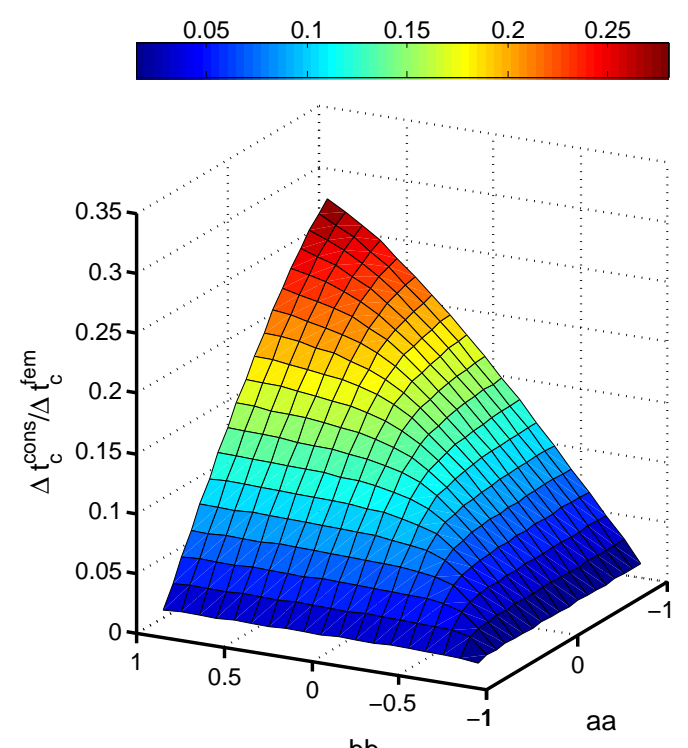

$\mathrm{bb}$

Fig. 16. Normalized critical time step for the lumped (left) and consistent (right) mass matrix as a function of the matter limit position: element cut by one segment, adjacent left and top edges.
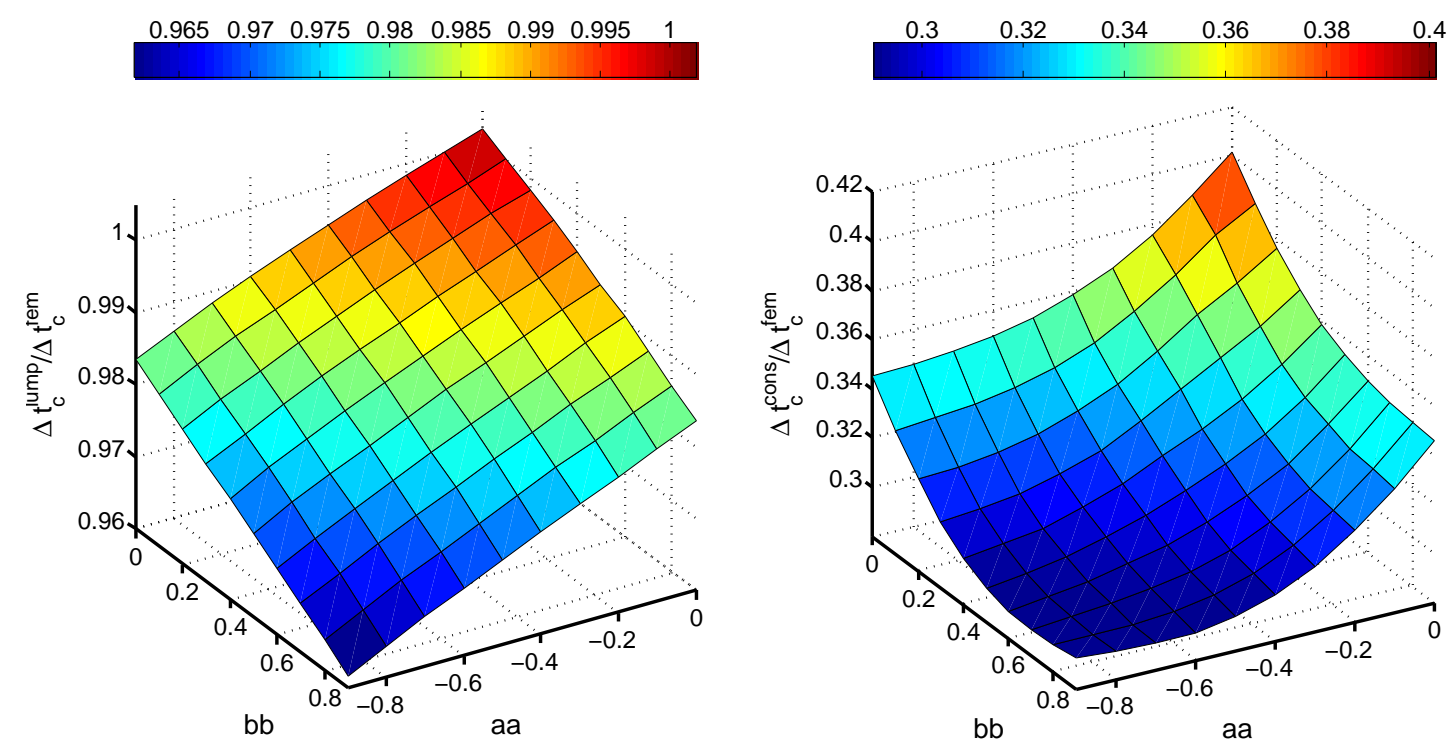

Fig. 17. Normalized critical time step for the lumped (left) and consistent (right) mass matrix as a function of the matter limit position: element cut by two segments.

In Figure 17, the element is cut by two segments, the intermediate point is the center of the element. The initial and final points are on adjacent edges and vary from the top right corner to the middle of the right edge and from the bottom left corner to the middle of the bottom edge. The critical time step is again lower with the consistent mass than with the lumped mass. Like in the previous case, the critical time step is minimum when the element is almost cut into two identical triangles $(a a \simeq-1, b b \simeq+1)$ and is maximum when the matter surface is maximum $(a a=0, b b=0)$. 
This last example shows that the key point for the minimum value of the critical time step is the volume fraction and not how the element is cut. For example, the maximum value obtained in this case with a volume fraction of $75 \%$ is the same as the one obtained in the first two cases with the same volume fraction. All the results for the minimum and maximum values for the critical time steps, as well as the corresponding volume fractions, considering consistent and lumped mass matrices, are given in Table 2.

\begin{tabular}{|c|c|c|c|c|}
\hline \multirow{2}{*}{} & \multicolumn{2}{|c|}{$\begin{array}{c}\text { Min value for } \\
\Delta t_{c}^{x-f e m} / \Delta t_{c}^{f e m} \\
\text { (vol. fraction) }\end{array}$} & \multicolumn{2}{|c|}{$\begin{array}{c}\text { Max value for } \\
\Delta t_{c}^{x-f e m} / \Delta t_{c}^{f e m}\end{array}$} \\
& $\begin{array}{c}\text { lumped } \\
\text { (vol. fraction) }\end{array}$ \\
\cline { 2 - 5 } & consistent & lumped & consistent \\
\hline opposite edges & $0.85(\sim 0 \%)$ & $0.1(\sim 0 \%)$ & $1(\sim 100 \%)$ & $0.5(\sim 100 \%)$ \\
\hline adjacent edges 1 & $0.97(\sim 50 \%)$ & $0.35(\sim 50 \%)$ & $1(\sim 100 \%)$ & $0.55(\sim 100 \%)$ \\
\hline adjacent edges 2 & $0.7(\sim 0 \%)$ & $0.05(\sim 0 \%)$ & $0.9(\sim 50 \%)$ & $0.25(\sim 50 \%)$ \\
\hline 2 segments & $0.965(\sim 50 \%)$ & $0.3(\sim 50 \%)$ & $1(\sim 75 \%)$ & $0.4(\sim 75 \%)$ \\
\hline
\end{tabular}

Table 2

Minimum and maximum values for the normalized critical time steps for hole enrichment and corresponding volume fraction.

Thus, the conclusion of this study can be focused on the first three results. With the proposed mass lumping expression for hole enrichment on piecewise bilinear quadrilateral, the critical time step is greater or equal than two-thirds of the critical time step of the same uncut element:

$$
\Delta t_{c \text { hole }}^{x-f e m} \geq \frac{2}{3} \Delta t_{c}^{f e m} .
$$

If the matter surface of the cut elements is at least half of the total surface of the element, the minimum value can be raised to $88 \%$ of the critical time step of the same uncut element.

\section{Numerical Examples}

All the examples presented in the following are treated under plain strain conditions. 

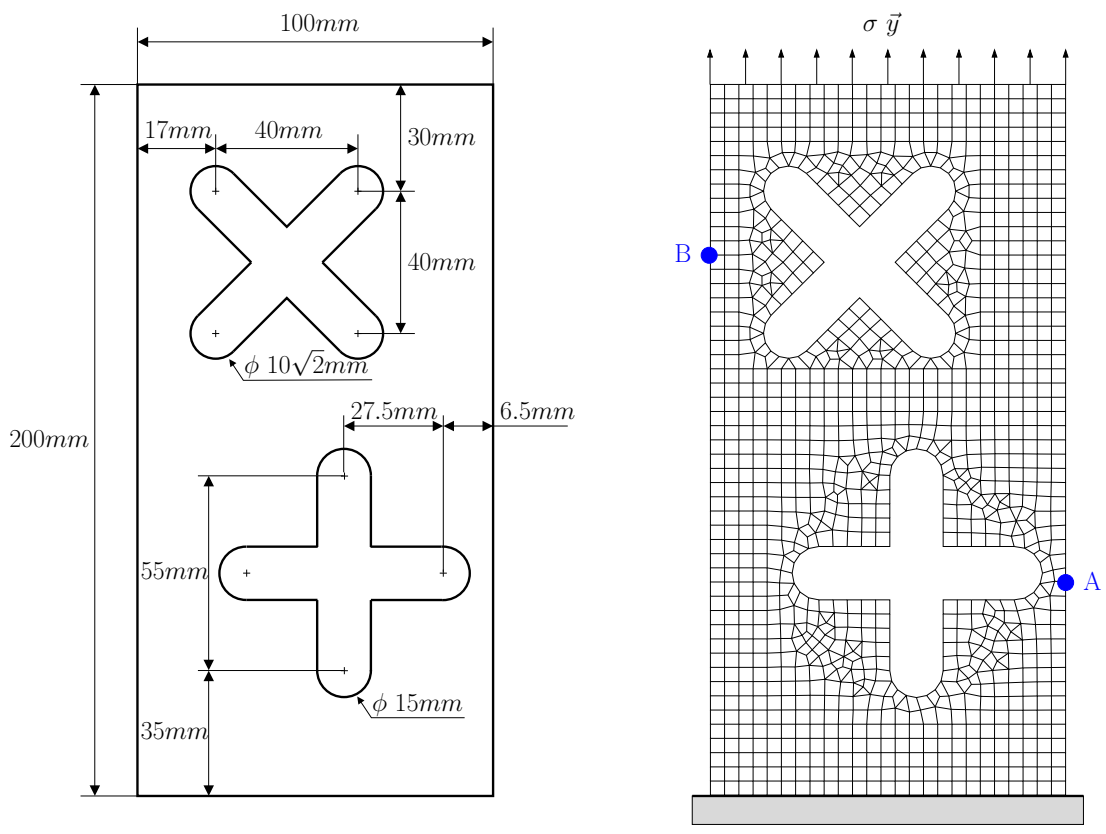

Fig. 18. Clamped plate with two cross-shaped holes: geometry, boundary conditions and structured conforming T3/Q4 FEM mesh.

\subsection{Rectangular plate with two cross-shaped holes}

The first example consists in a rectangular structure with two cross-shaped holes clamped on one side and submitted to a sudden vertical stress on the other side. The geometry can be observed in Figure 18. This example is quite complex as the shape of the two holes are difficult to represent with a relatively constant mesh density. A structured conforming Q4/T3 finite element mesh with an almost constant mesh density can be observed in Figure 18. The corresponding X-FEM mesh is a structured one composed of $49 \times 49$ quadrilateral elements. The specimen is made of steel with the following material parameters: $E=2.1 \times 10^{11} \mathrm{~Pa}, \nu=0.3, \rho=7800 \mathrm{~kg} \cdot \mathrm{m}^{-3}$, the applied stress is $\sigma=10^{6} \mathrm{~Pa}$, the total time of the simulation is $3 \mathrm{~ms}$. The critical time step for the finite element mesh is $\Delta t_{c}^{f e m}=0.22 \mu s$, which corresponds to a simulation of 13636 time steps. The critical time step of the X-FEM mesh without the hole is $\Delta t_{c \text { no hole }}^{x-f e m}=0.664 \mu \mathrm{s}$. The mesh is cut by the hole in a way such that a ratio of around $90 \%$ is acceptable, therefore the critical time step is taken to be $\Delta t_{c}^{x-f e m}=0.6 \mu s$, which corresponds to a simulation of 5000 time steps. This corresponds to one third of the number of time steps needed for the finite element calculation.

Remark 3 If the "worst case" ratio of $2 / 3$ was chosen, the critical time step for the X-FEM mesh would be $\Delta t_{c}^{x-f e m}=0.442 \mu \mathrm{s}$ which corresponds to a simulation of 6788 time steps. It is worth noting that even in this case, we only need about half the number of time steps compared to the equivalent finite 


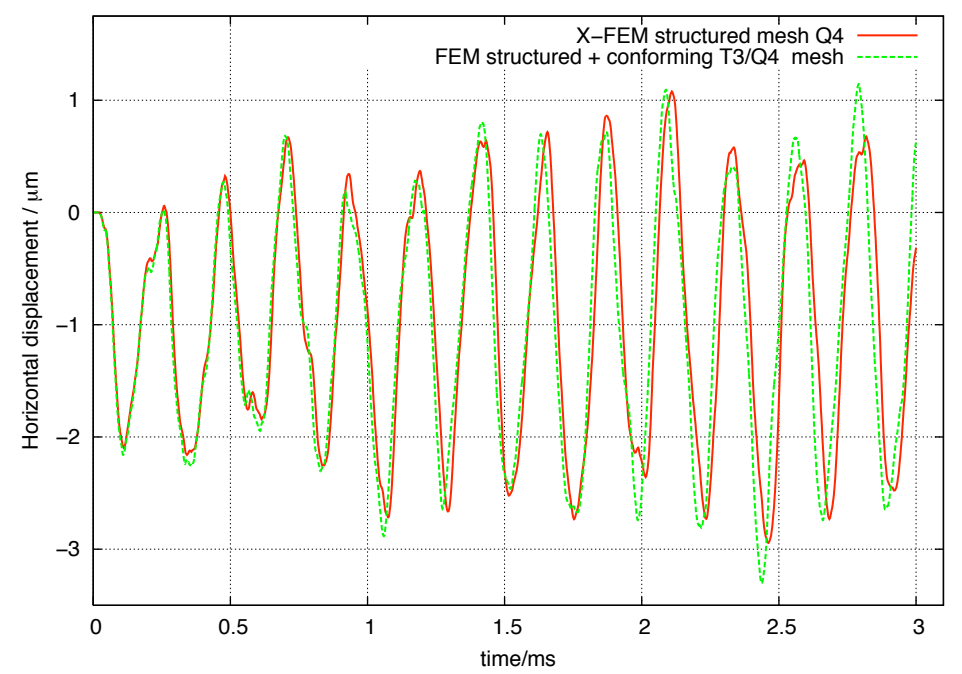

Fig. 19. Clamped plate with two cross-shaped holes: horizontal displacement at point A

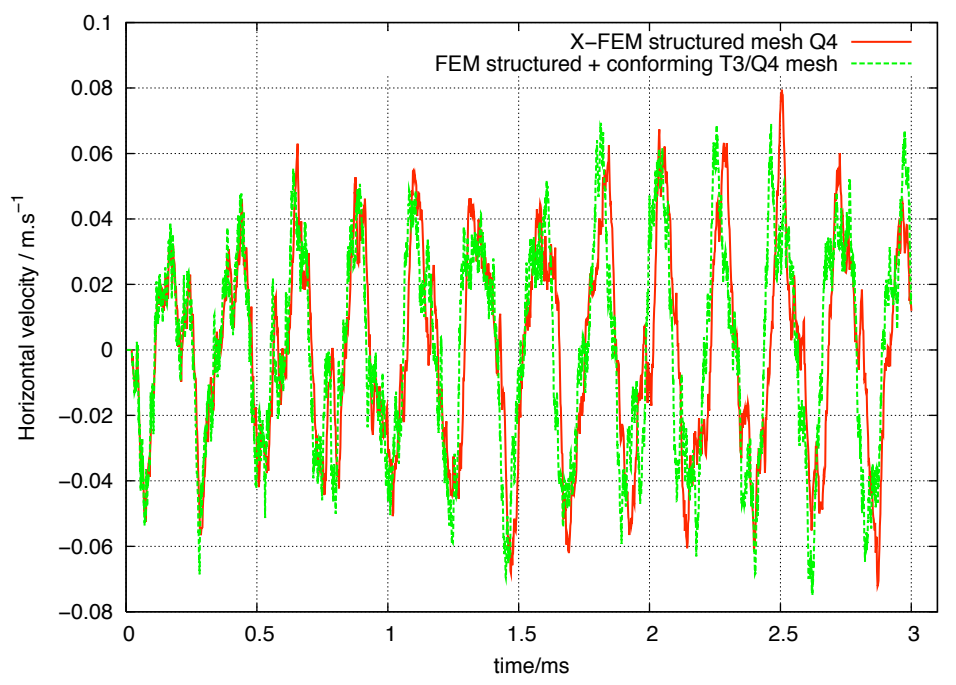

Fig. 20. Clamped plate with two cross-shaped holes: horizontal velocity at point A

element mesh.

The results at point A are given in Figure 19 for the horizontal displacement and in Figure 20 for the horizontal velocity. The results at point $\mathrm{B}$ are given in Figure 21 for the vertical displacement and in Figure 22 for the vertical velocity. In all the cases the two results compare quite well. This shows that the proposed method allows us to obtain the same accuracy with X-FEM with a number of time step between $33 \%$ and $50 \%$ of the one needed for a finite element calculation. 


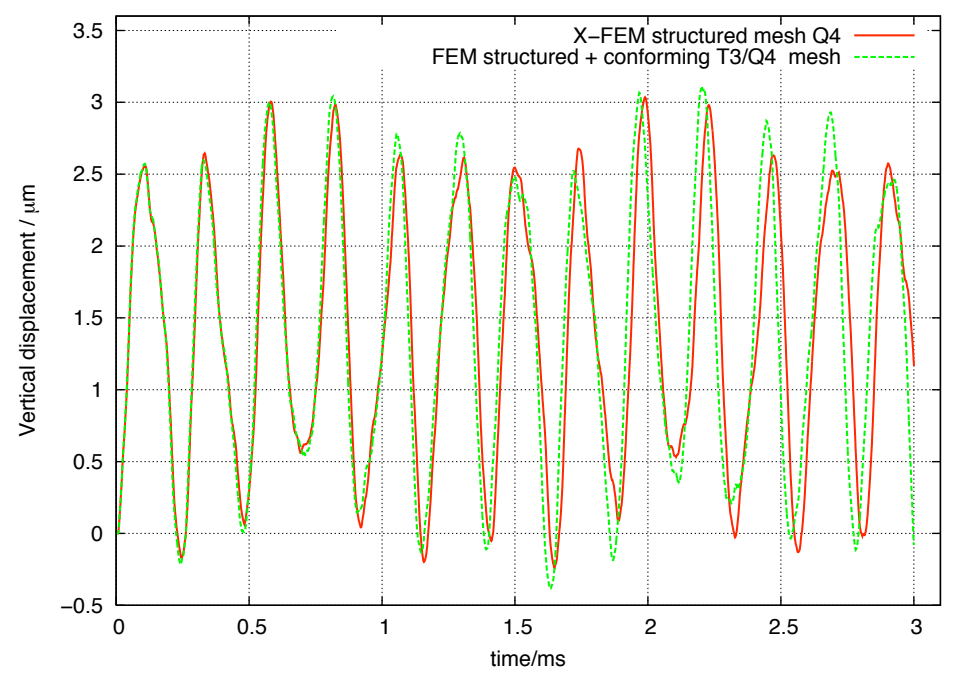

Fig. 21. Clamped plate with two cross-shaped holes: vertical displacement at point $\mathrm{B}$

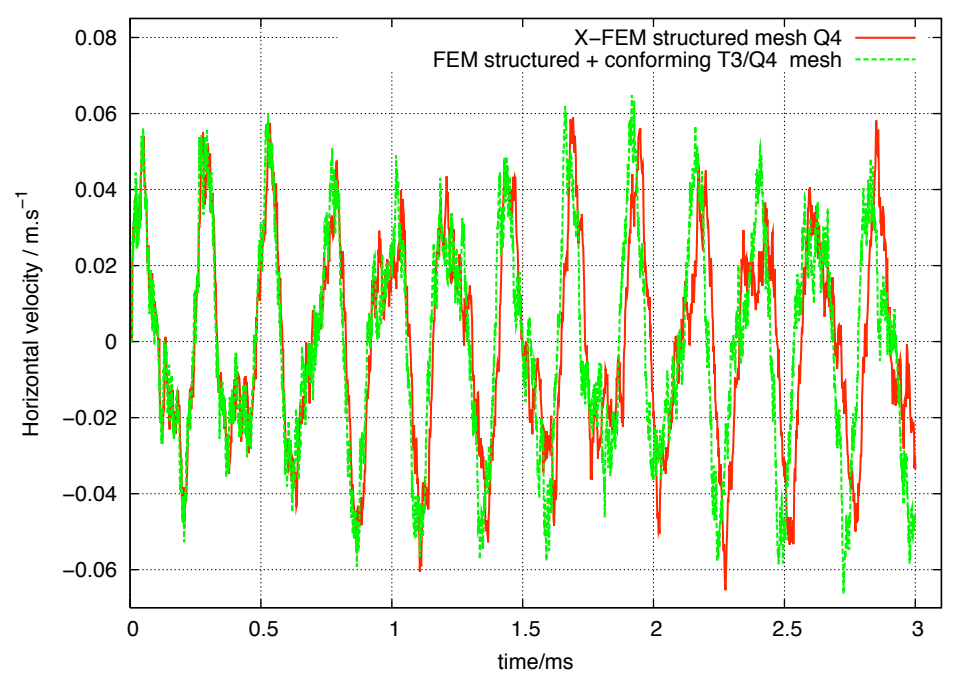

Fig. 22. Clamped plate with two cross-shaped holes: vertical velocity at point B

\subsection{Stationary and moving semi-infinite cracks}

\subsubsection{Stationary mode I semi-infinite crack}

We consider as a first example in fracture mechanics the case of a semi-infinite mode I crack in an infinite medium submitted to a tensile stress wave. The analytical solution for the mode I dynamic stress intensity factor was obtained by Freund [12]. We consider the finite geometry given in Figure 23, therefore we can only compare the results with the analytical solution until the tensile stress wave is reflected on the bottom side and reaches again the crack tip. 


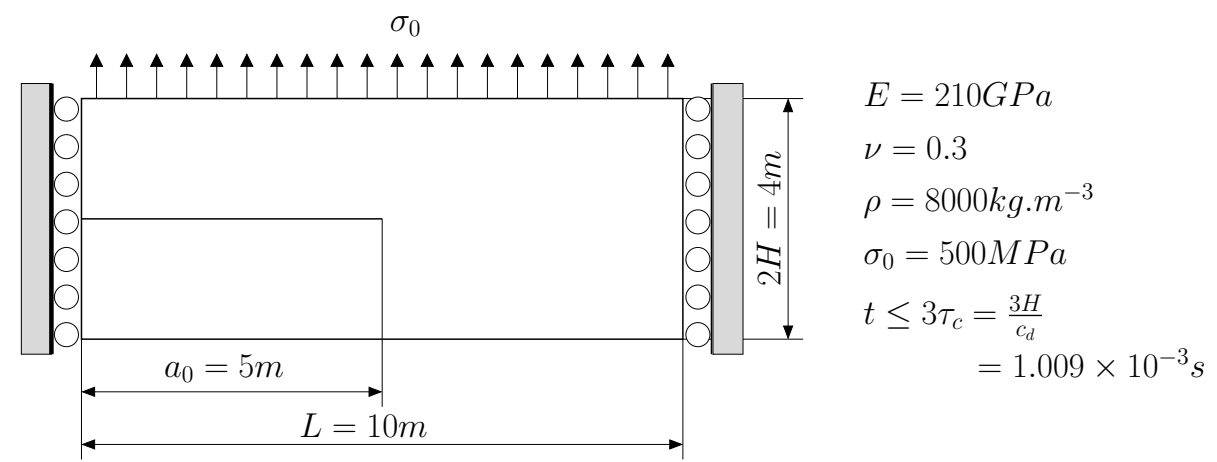

Fig. 23. Mode I semi-infinite crack problem: finite geometry and material parameters.

The time needed by the stress wave to reach the crack tip for the first time is $\tau_{c}=\frac{H}{c_{d}}$, where $c_{d}$ is the dilatational wave speed. The total simulation is therefore limited to $t \leq 3 \tau_{c}=1.009 \times 10^{-3} s$ with the material parameters given in Figure 23.

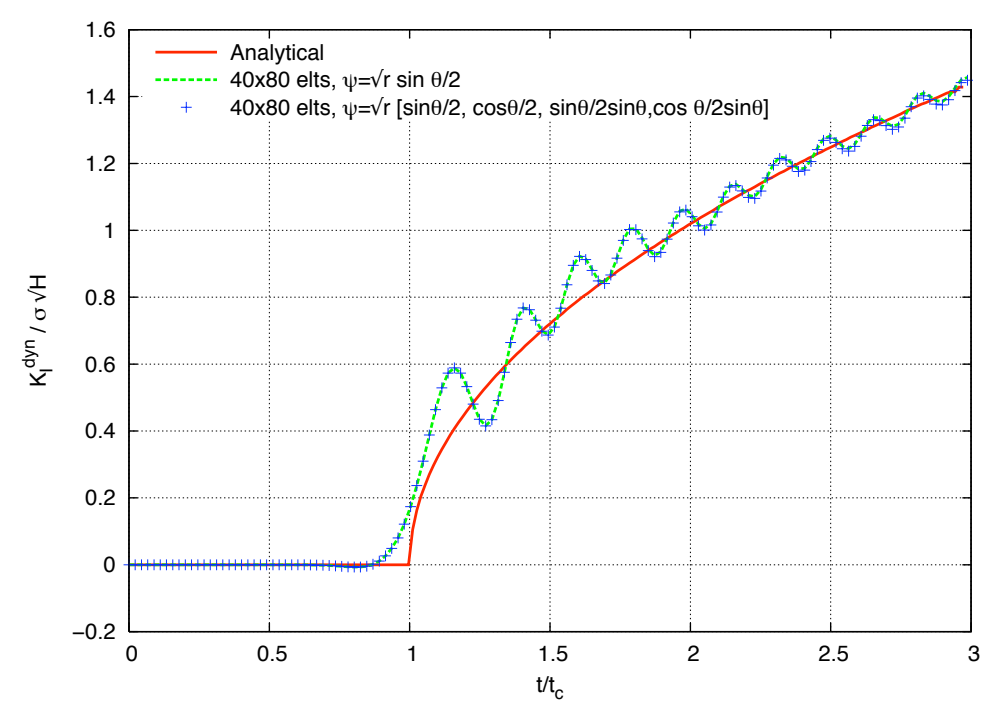

Fig. 24. Normalized mode I stress intensity factor vs. normalized time for a stationary semi-infinite crack: analytical solution, $40 \times 80$ elements mesh with reduced enrichment basis, $40 \times 80$ elements mesh with complete enrichment basis.

As the wave reaches the crack tip, the mode I dynamic stress intensity factor for a stationary crack is given by:

$$
K_{I}^{d y n}(0, t)=\frac{2 \sigma_{0}}{1-\mu} \sqrt{\frac{c_{d} t(1-2 \mu)}{\pi}},
$$

where $\mu$ is the second Lamé constant. For a moving crack tip, the mode I dynamic stress intensity factor is given by:

$$
K_{I}^{d y n}(\dot{a}, t)=k(\dot{a}) K_{I}^{d y n}(0, t)
$$




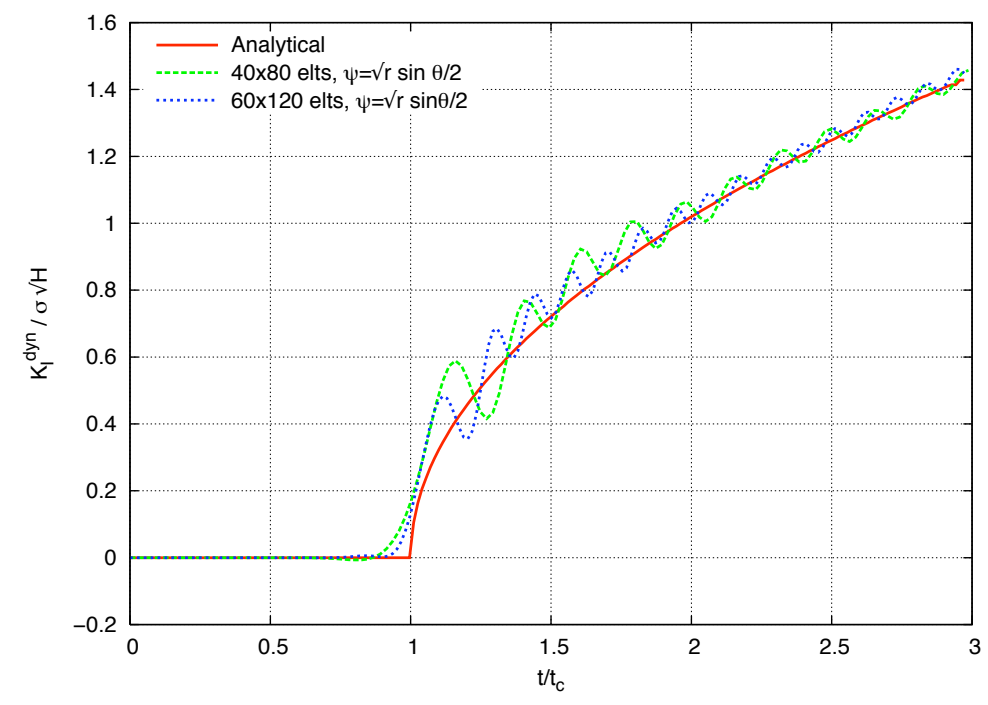

Fig. 25. Normalized mode I stress intensity factor vs. normalized time for a stationary semi-infinite crack: analytical solution, $40 \times 80$ elements mesh with reduced enrichment basis, $60 \times 120$ elements mesh with reduced enrichment basis.

where $k$ is a universal function of the crack tip speed $\dot{a} . k$ can be approximated by the following expression:

$$
k(\dot{a})=\frac{1-\dot{a} / c_{r}}{1-\dot{a} / 2 c_{r}}
$$

where $c_{r}$ is the Rayleigh wave speed. Finally, one can write:

$$
K_{I}^{d y n}(\dot{a}, t)=\frac{2 \sigma_{0}}{1-\mu} \sqrt{\frac{c_{d} t(1-2 \mu)}{\pi}} \frac{1-\dot{a} / c_{r}}{1-\dot{a} / 2 c_{r}} .
$$

We first use a mesh of $40 \times 80$ quadrilateral elements with a critical time step of $\Delta t_{c}^{x-f e m}=\Delta t_{c}^{f e m} / 2=7.5 \mu \mathrm{s}$, that is a simulation of 134 time steps. We compare the normalized mode I dynamic stress intensity factor $K_{I}^{d y n} / \sigma_{0} \sqrt{H}$ as a function of the normalized time $t / \tau_{c}$ obtained with the proposed method with the analytical solution.

The first result shown in Figure 24 compares the results obtained with only one singular enrichment function $\psi=\sqrt{r} \sin \frac{\theta}{2}$ and the results obtained with the complete singular enrichment basis $\psi=\sqrt{r}\left[\sin \frac{\theta}{2}, \cos \frac{\theta}{2}, \sin \frac{\theta}{2} \sin \theta, \cos \frac{\theta}{2} \sin \theta\right]$. We can observe that the results are almost identical and compare well to the analytical solution. The solution presents some oscillations that were also obtained in Menouillard et al. [24] with a purely discontinuous X-FEM explicit approach. This result demonstrates that only the first singular enrichment function is sufficient in order to obtain a good accuracy for a mode I stationary crack. 
The same calculation was also performed with one enrichment function with a mesh of $60 \times 120$ quadrilateral elements with a critical time step of $\Delta t_{c}^{x-f e m}=$ $\Delta t_{c}^{f e m} / 2=5 \mu s$, that is a simulation of 200 time steps. In Figure 25, we compare the results obtained with this mesh and the previous coarser one. Again, the results compare well with the analytical solution, the finer mesh still produces oscillations but with a much lower amplitude. This result shows the good convergence of the method with the mesh size in terms of the dynamic mode I stress intensity factor.

\subsubsection{Moving mode I semi-infinite crack}

As in Duarte et al. [9], Belytschko et al. [3], Chen et al. [2], Réthoré et al. [33, 34] and Menouillard et al. [24], we now consider the case of stationary then moving crack. The crack remains stationary for $0 \leq t<1.5 \tau_{c}$ then propagates in mode I at a constant velocity $\dot{a}=1500 \mathrm{~m}_{\mathrm{s}} \mathrm{s}^{-1}$ for $t \geq 1.5 \tau_{c}$.

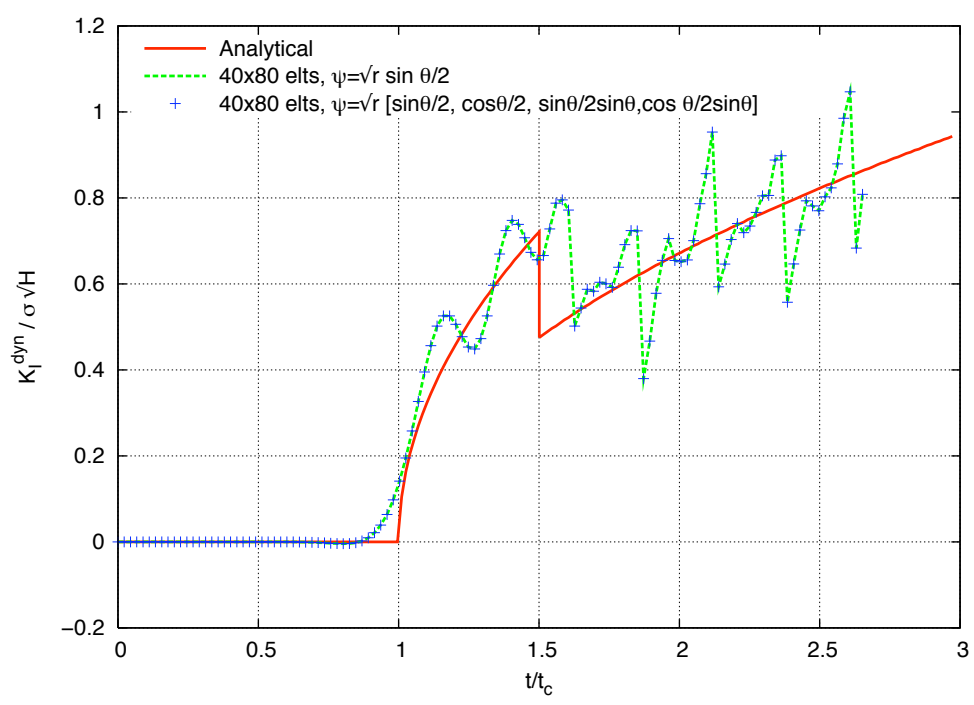

Fig. 26. Normalized mode I stress intensity factor vs. normalized time for a stationary then moving semi-infinite crack: analytical solution, $40 \times 80$ elements mesh with reduced enrichment basis, $40 \times 80$ elements mesh with complete enrichment basis.

We consider the same two cases as in the previous example. In Figure 26, we compare the results obtained with a mesh composed of $40 \times 80$ quadrilateral elements with a critical time step of $\Delta t_{c}^{x-f e m}=\Delta t_{c}^{f e m} / 2=7.5 \mu s$ with only one singular enrichment function and with the complete singular enrichment basis. The results compare relatively well with the analytical solution and do not show any visible difference between the two enrichment basis used. The numerical solution presents oscillations when the crack is moving as it is the case with other X-FEM explicit dynamics techniques presented in Belytschko et al. [3], Zi et al. [39] and Menouillard et al. [24]. It is interesting to note here, that we did not apply any numerical filter to the results obtained with the proposed method contrary to the ones presented in the References cited 


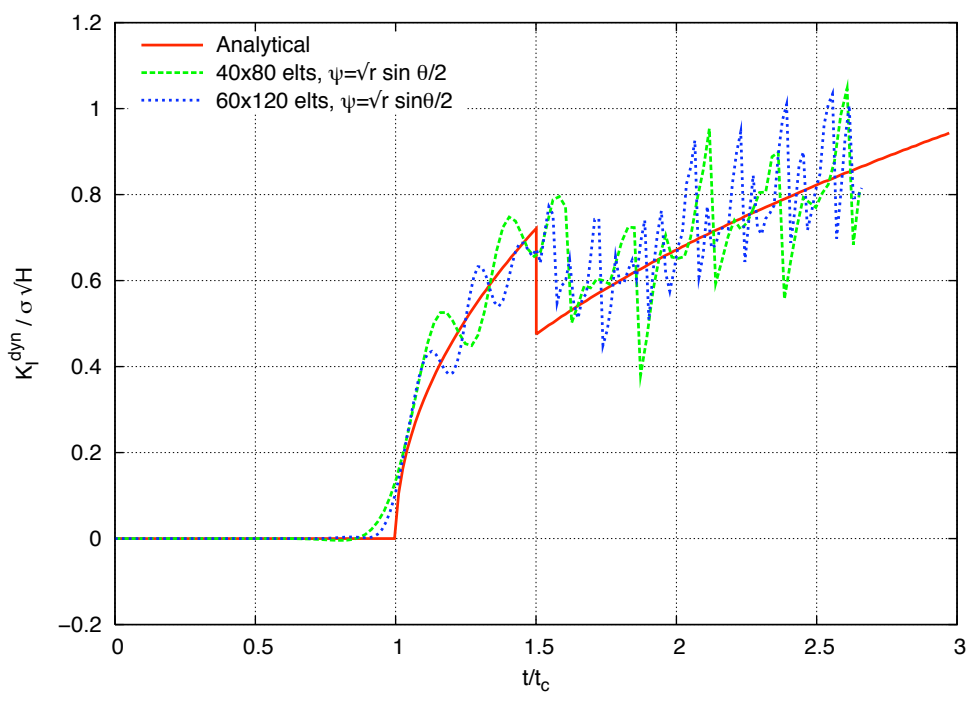

Fig. 27. Normalized mode I stress intensity factor vs. normalized time for a stationary then moving semi-infinite crack: analytical solution, $40 \times 80$ elements mesh with reduced enrichment basis, $60 \times 120$ elements mesh with reduced enrichment basis.

above. This indicates that the accuracy obtained with the proposed method is slightly better for this example.

In Figure 27, we compare the results obtained with only one singular enrichment function with the $40 \times 80$ and $60 \times 120$ elements meshes. The finer mesh computed with a smaller time step allows us to obtain a more accurate localization of the crack initiation in time. The results presents similar oscillations but with a lower amplitude.

\subsubsection{Stationary mixed mode semi-infinite crack}

We now consider the case of a stationary mixed mode semi-infinite crack. This example was also treated in Chen et al. [2] and Réthoré et al. [34] and an analytical solution was obtained by Lee and Freund [22]. We use the finite geometry given in Figure 28, therefore we can only compare the results with the analytical solution until the compressive stress wave is reflected on the right edge and reaches again the crack tip. The time needed by the stress wave to reach the crack tip for the first time is $\tau_{c}=\frac{a_{0}}{c_{d}}$, where $c_{d}$ is the dilatational wave speed. The total simulation is therefore limited to $t \leq 3 \tau_{c}=542 \mu \mathrm{s}$ with the material parameters given in Figure 28.

We compare the normalized dynamic stress intensity factor

$$
\bar{K}^{d y n}(t)=\frac{K^{d y n}(t)}{-E V_{0} \sqrt{a_{0}} /\left(2\left(1-\nu^{2}\right) c_{d} \sqrt{\pi}\right)}
$$

as a function of the normalized time $t / \tau_{c}$ obtained with the proposed method 


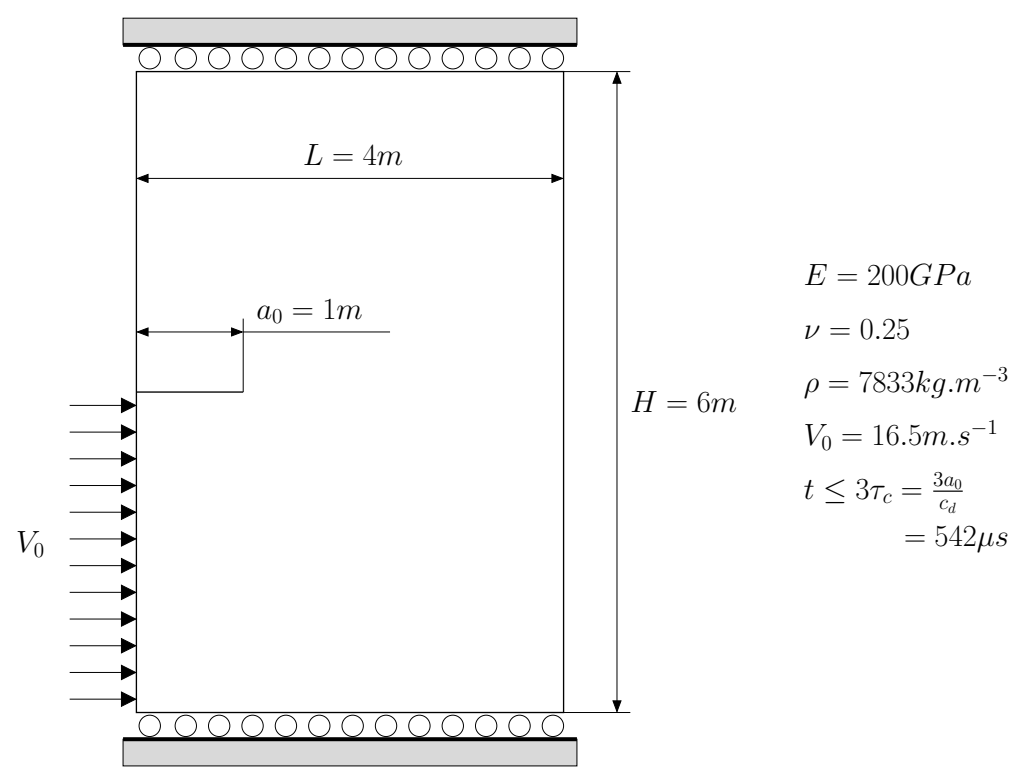

Fig. 28. Mode II semi-infinite crack problem: finite geometry and material parameters.

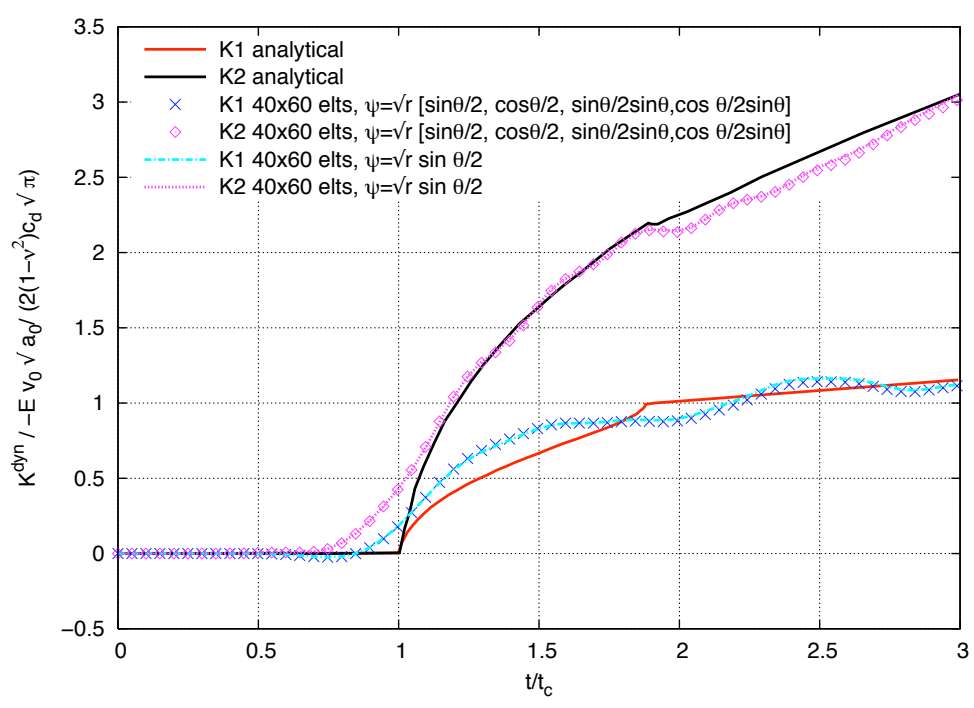

Fig. 29. Normalized stress intensity factors vs. normalized time for a stationary semi-infinite mixed mode crack: analytical solution, $40 \times 60$ elements mesh with complete enrichment basis, $40 \times 60$ elements mesh with reduced enrichment basis.

with the analytical solution.

We first use a mesh of $40 \times 60$ piecewise bilinear quadrilateral elements with a critical time step of $\Delta t_{c}^{x-f e m}=\Delta t_{c}^{f e m} / 2=9 \mu \mathrm{s}$. In Figure 29, we plot the results obtained with one singular enrichment function and with the complete singular enrichment basis. We can observe that those two results are almost identical and compare quite well with the analytical one. This shows that only one singular enrichment function is also sufficient to obtain a good accuracy in mixed mode. 


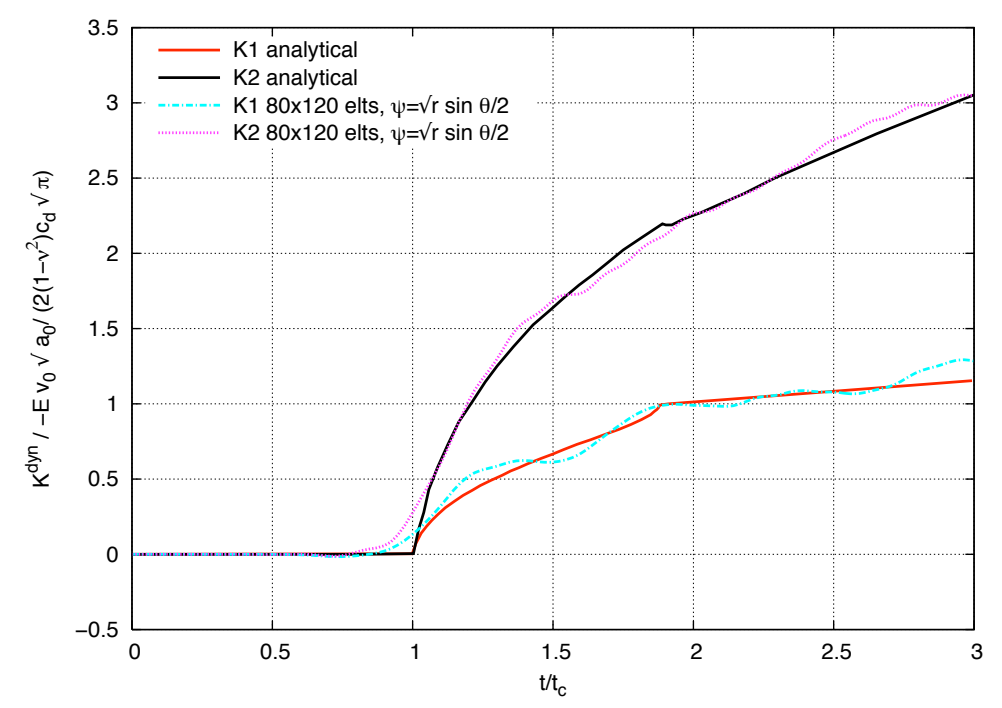

Fig. 30. Normalized stress intensity factors vs. normalized time for a stationary semi-infinite mixed mode crack: analytical solution, $80 \times 120$ elements mesh with reduced enrichment basis.

In Figure 30, we plot the results obtained with only one singular enrichment for a mesh of $80 \times 120$ elements. The calculation was performed with a critical time step of $\Delta t_{c}^{x-f e m}=\Delta t_{c}^{f e m} / 2=4.5 \mu \mathrm{s}$. We can observe that the results still present a few oscillations but with a lower amplitude. These results show the good convergence of the method with the mesh size in terms of the dynamic stress intensity factors.

\subsection{Kalthoff's experiment}

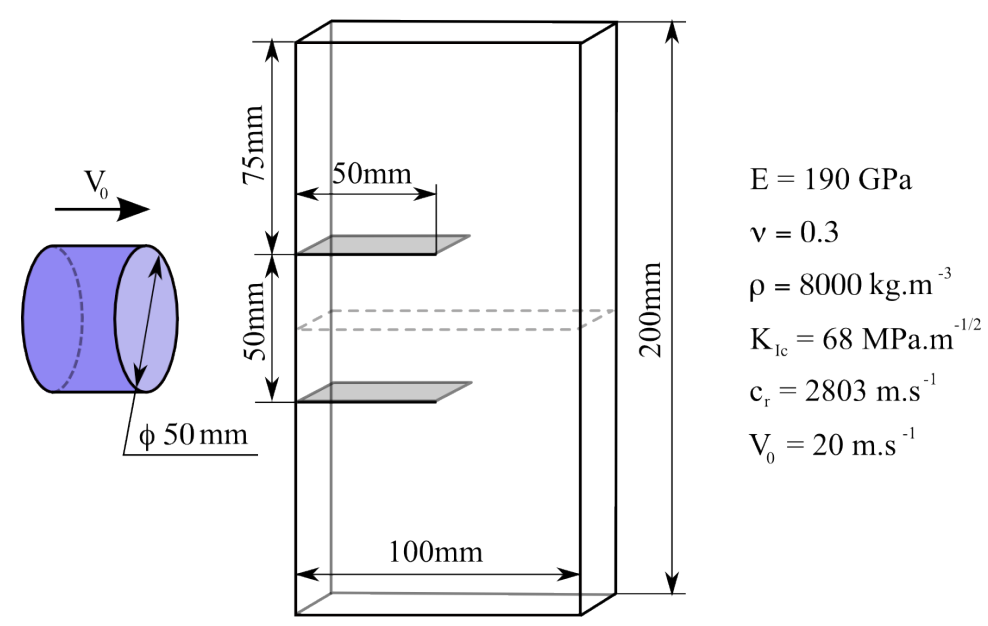

Fig. 31. Experimental setup and geometry for Kalthoff's experiment.

This example deals with the numerical simulation of Kalthoff's experiment of the failure mode transition under pure mode II loading [19]. The experimental configuration is presented in Figure 31: a plate with two symmetrical edge 
cracks is impacted by a projectile at a given speed $V_{0}$. By modifying the projectile's velocity, Kalthoff observed a transition in the type of failure. At low velocity, i.e. under low strain rate, brittle failure is observed with a simultaneous propagation of the two cracks with a global angle between $60^{\circ}$ and $70^{\circ}$. If one increases the projectile's speed, a transition between brittle fracture and shear band propagation (with a propagation angle of approximatively $-10^{\circ}$ ) occurs. We consider here only the brittle propagation with an impact velocity of 20m.s $\mathrm{s}^{-1}$. As in Belytschko et al. [3], Zi et al. [39] and Menouillard et al. [24], we model the experiment in plane strain and consider only the upper half of the plate with the appropriate symmetry boundary conditions. The material properties are those of a 18Ni1900 maraging type steel and are given in Figure 31. A mesh of $80 \times 80$ piecewise bilinear quadrilateral elements was used, with a critical time step of $\Delta t_{c}^{x-f e m}=\Delta t_{c}^{f e m} / 2=0.11 \mu \mathrm{s}$. The simulation time was $100 \mu$ s represented by 900 time steps.
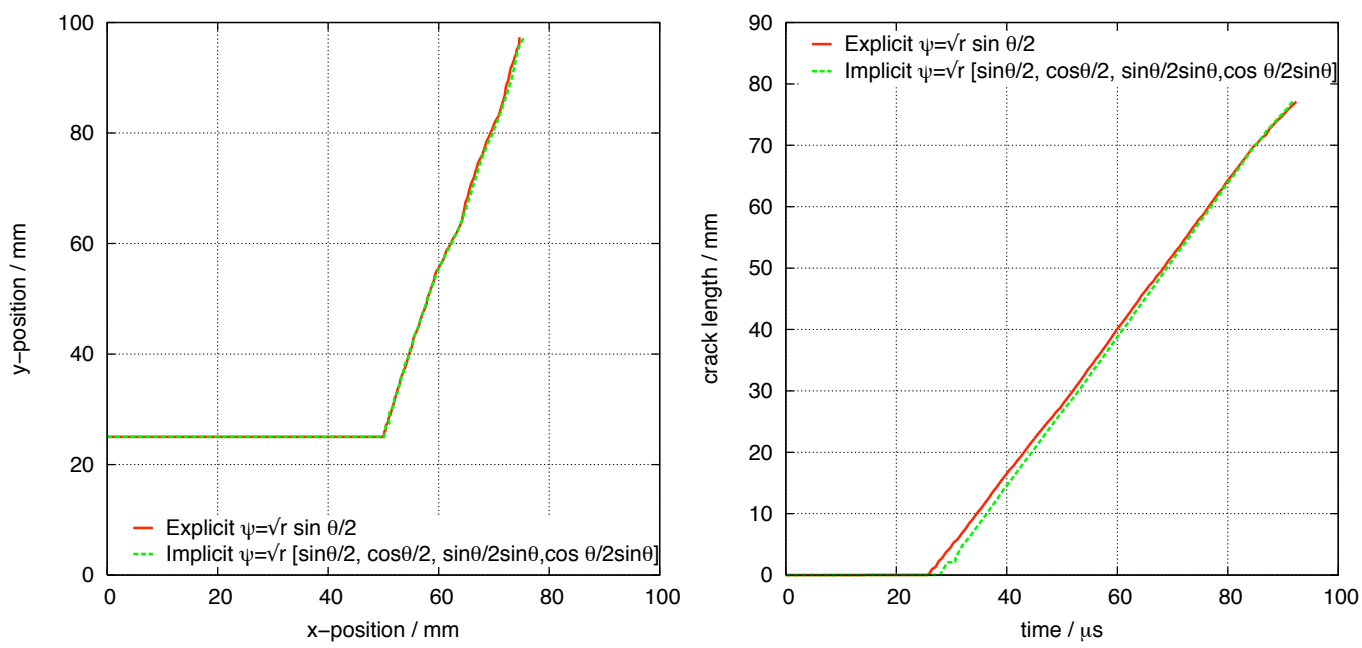

Fig. 32. Kalthoff's experiment, results for the proposed method and for an implicit X-FEM calculation (from [24]): final crack path (left) and crack length versus time (right).

The left part of Figure 32 shows the crack path obtained with the proposed method and with an X-FEM implicit mean acceleration time integrator with a full singular enrichment basis with the same mesh (from Menouillard et al. [24]). These results are very similar and the overall angle is in good agreement with the experimental and previously published numerical results. Looking at the details of the crack path, we observe the same phenomena as in Menouillard et al. [24] and de Borst et al. [8]. The crack starts to propagate with an angle of $65^{\circ}$ at $26 \mu \mathrm{s}$; then a small deviation is observed around $50 \mu \mathrm{s}$ and the crack continues to propagate with an angle of $65^{\circ}$ from $65 \mu \mathrm{s}$. This deviation is related to the successive reflections of the compressive stress wave. The wave travels from the left edge to the right one when the projectile hits the plate. Then the wave is reflected on the right free surface as a tensile wave, reaches the crack tip around $26 \mu s$ and the propagation starts. This tensile wave travels from the crack tip to the left free surface and is again reflected as 

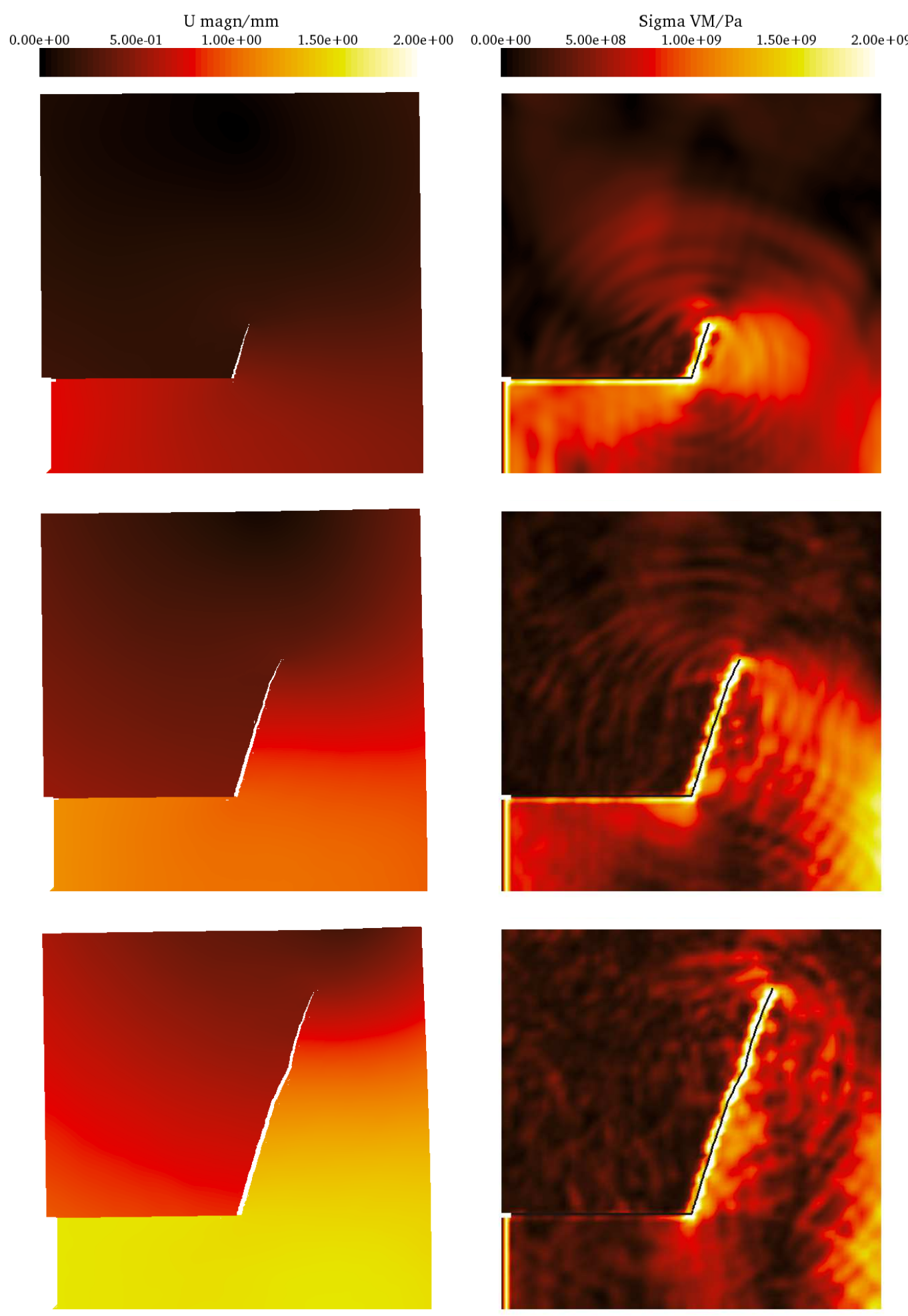

Fig. 33. Kalthoff's experiment: results at $40 \mu s, 60 \mu s$ and $80 \mu s$. Left: displacement magnitude on the deformed configuration (amplified 2 times), right: Von Mises stress field on the initial configuration. 
a compressive wave. Then, this wave travels in the right direction and reaches the moving crack tip around $50 \mu \mathrm{s}$. This compressive wave induces the crack deviation, but is subsequently reflected against the right edge as a tensile wave which finally reaches the crack tip at $65 \mu \mathrm{s}$, after which the crack continues to propagate with the initial angle.

We also plot on the right part of Figure 32 the crack length as a function of time for the two methods. We can also observe that the two results are almost identical, although the crack seams to propagate a little earlier and with a slightly higher velocity with the explicit scheme than with the implicit one. Finally we plot in Figure 33 the displacement magnitude on the deformed configuration (amplified two times) and the Von Mises stress field on the initial configuration at various times. We can observe on these plots the crack deviation and wave reflections that were noted above.

\subsection{Compact Compression Specimen}

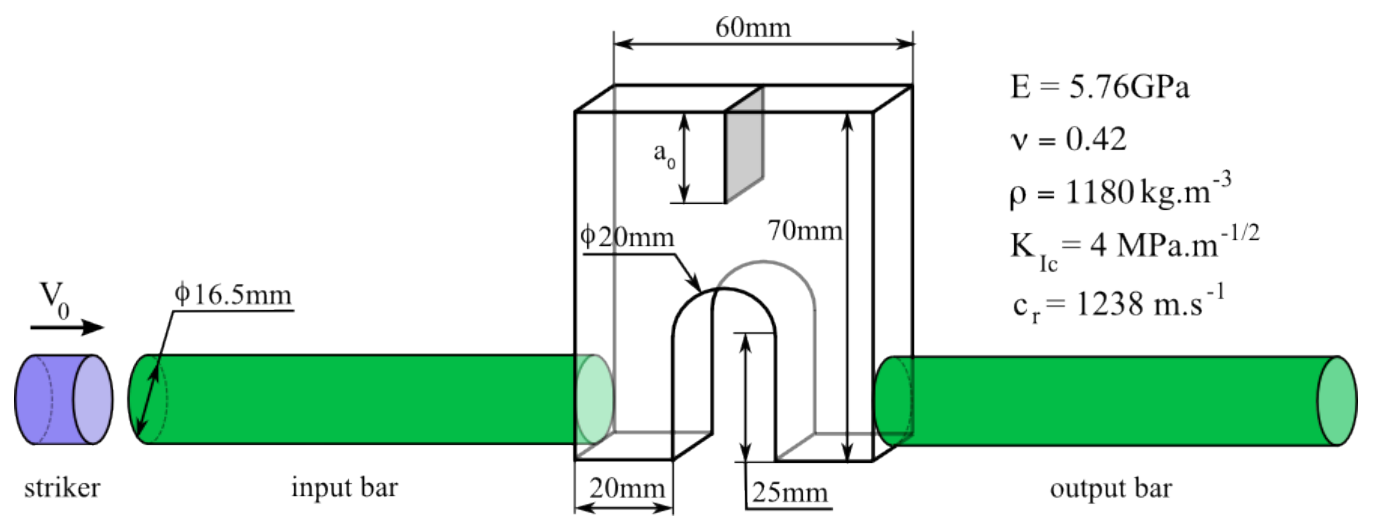

Fig. 34. Experimental setup and geometry for the Compact Compression Specimen experiment.

The last example deals with the Compact Compression Specimen experiment first introduced by Bui et al. [6] and further developed by Maigre and Rittel [23]. The interest of the CCS experiment in our case is that there is always a mixed-mode loading condition at the crack tip. The test rig is a Split Hopkinson Pressure Bar (Kolsky bar), the experimental setup is described in Figure 34. As in Seelig et al. [36] and Menouillard et al. [24], the input bar is modelled by the experimental dynamic load. Since during the simulation time of $150 \mu \mathrm{s}$ the stress wave does not reflect on the surface between the specimen and the output bar, a free surface boundary condition is used instead of the output bar. The material properties are those of PMMA and are given in Figure 34, the initial crack length is $a_{0}=18.3 \mathrm{~mm}$.

A rectangular mesh of $60 \times 41$ piecewise bilinear quadrilateral elements was used. The void region in the center of the specimen was modelled as a hole. 
The critical time step due to the presence of the crack is lower than the one due to the modelling of the hole. Thus, we used the rule developed in paragraph 3.2 , the critical time step is $\Delta t_{c}^{x-f e m}=\Delta t_{c}^{f e m} / 2=0.15 \mu s$, and we used 1000 time steps for the simulation.

We plot in Figure 35 the numerical crack path and the crack length as a function of time obtained with the proposed method and with an X-FEM implicit mean acceleration time integrator with the same mesh (from Menouillard et al. [24]). The results are very similar and agree well with the experimental results. This last example is very interesting as it demonstrates the performance of the method: a complex experiment was modelled by a simple rectangular mesh and free surfaces and moving discontinuities were represented accurately by the X-FEM.
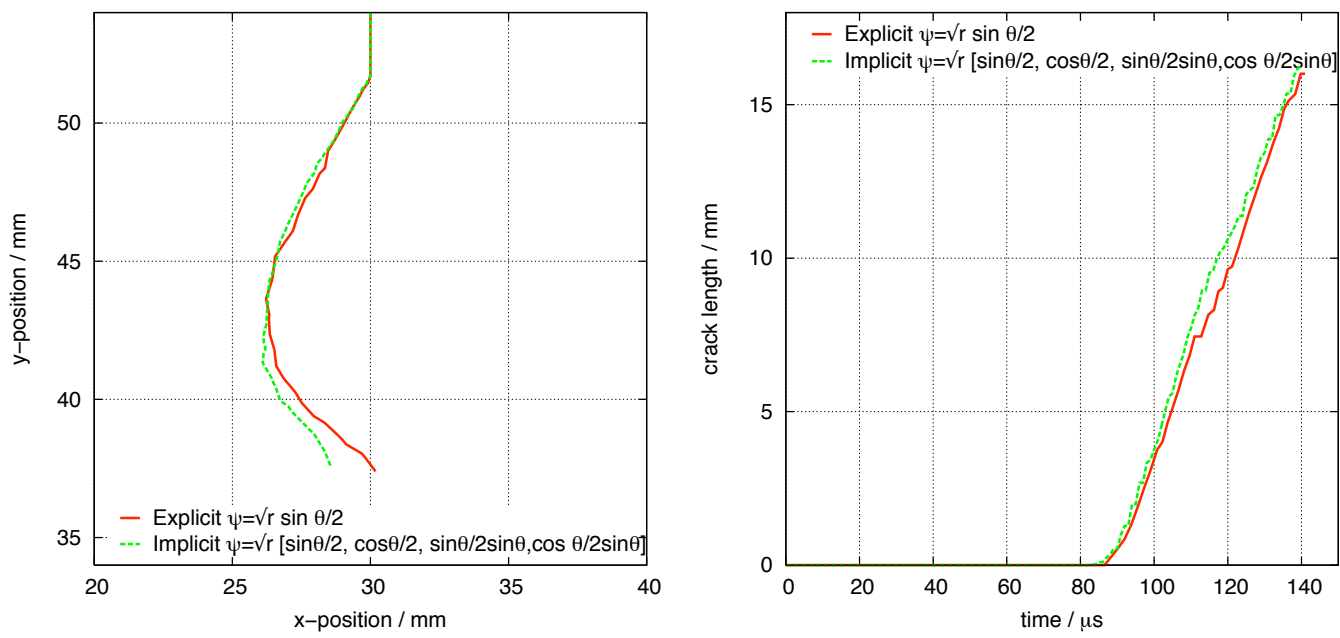

Fig. 35. Compact Compression Specimen, results for the proposed method and for an implicit X-FEM calculation (from [24]): final crack path (left) and crack length versus time (right).

\section{Conclusions}

We have presented in this paper a general expression for the lumped mass in the eXtended Finite Element Method for any enrichment function. This expression is based on the exact representation of the kinetic energy of the rigid body modes and enrichment modes. We focused our study on cracks, free boundaries and holes modelling with X-FEM. We showed that the proposed lumping formula is a generalization of some lumping techniques that were proposed for discontinuous enrichment for cracks (see [24, 25]) and for free boundaries for constant strain elements (see [35]).

We focused our study on singular enrichment for cracks and free boundaries with quadrilateral elements for which lumping techniques are proposed for the 
first time. A detailed analysis of critical time steps estimates was performed for these two cases and simple rules were developed. Several numerical examples were shown to validate the method. For free boundaries, these results compare well with finite elements but have a much higher critical time step for a given mesh density. For cracks modelling, we obtained almost identical results to previously published ones considering an unconditionally stable implicit time integrator. The next step, as pointed out in the introduction, is to extend this approach to three-dimensional dynamic crack modelling for elastic and elastic-plastic cases and to compare those results with experiments developed in the laboratory.

\section{Acknowledgements}

This work was supported by the French Agence Nationale pour la Recherche and Centre National de la Recherche Scientifique under contract ANR-CNRS DYNRUPT JC05_45254. This support is gratefully acknowledged. We would also like to thank Drs Réthoré and Menouillard for providing their results for the last two examples.

\section{A Fracture parameters and criteria}

\section{A.1 Fracture parameters}

The interaction integral, first proposed in the static case by Moran and Shih [28] and Gosz et al. [13], and subsequently extended to the dynamic case (see, e.g., Krysl and Belytschko [21] and Réthoré et al. [34]), is used for the separation of the mixed-mode SIFs. The separation is obtained via a twofield problem consisting of the actual fields $(\mathbf{u}, \boldsymbol{\sigma})$ and the auxiliary fields $\left(\mathbf{u}^{\text {aux }}, \boldsymbol{\sigma}^{\text {aux }}\right)$. Then, the Lagrangian conservation law for a virtual crack extension field $\mathbf{q}$ leads to the following expression of the domain-independent dynamic interaction integral $I^{\text {int }}$ :

$$
\begin{aligned}
I^{i n t}= & -\int_{A} q_{k, j}\left[\left(\sigma_{m l}^{a u x} u_{m, l}-\rho \dot{u}_{l} \dot{u}_{l}^{a u x}\right) \delta_{k j}-\left(\sigma_{i j}^{a u x} u_{i, k}+\sigma_{i j} u_{i, k}^{a u x}\right)\right] d S \\
& +\int_{A} q_{k}\left[\left(\sigma_{i j, j}^{a u x} u_{i, k}+\rho \ddot{u}_{i} u_{i, k}^{a u x}\right)+\left(\rho \dot{u}_{i}^{a u x} \dot{u}_{i, k}+\rho \dot{u}_{i} \dot{u}_{i, k}^{a u x}\right)\right] d S
\end{aligned}
$$

where $A$ is the area delimited by any contour $\partial A$ enclosing the crack tip. $\mathbf{q}$ is the virtual crack extension field and is taken to be tangent to the crack faces, with a unit norm at the crack tip $(\|\mathbf{q}\|=1)$ and a zero norm outside $A$ $(\|\mathbf{q}\|=0)$ as presented in Figure A.1. 

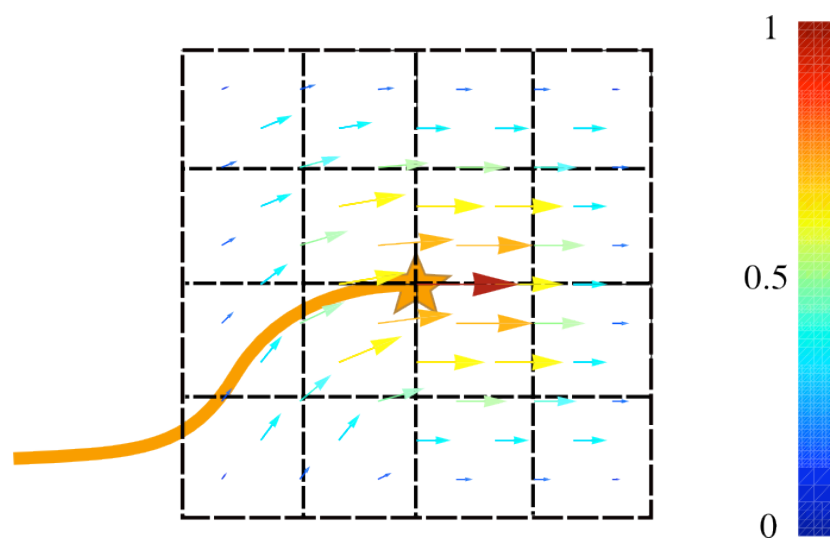

Fig. A.1. Virtual crack extension field tangent to the crack faces for a curved crack.

Then, a dynamic energy analysis provides an equivalent to Irwin's relation in plane strain:

$$
I^{i n t}=\frac{2\left(1-\nu^{2}\right)}{E}\left(f_{1}(\dot{a}) K_{I}^{d y n} K_{I}^{a u x}+f_{2}(\dot{a}) K_{I I}^{d y n} K_{I I}^{a u x}\right),
$$

where $K_{I}^{a u x}$ and $K_{I I}^{a u x}$ are the stress intensity factors of the auxiliary fields, $f_{i}$ are universal functions of the velocity of the crack tip $\dot{a}$ :

$$
\begin{aligned}
& f_{i}(\dot{a})=\frac{\beta_{i}\left(1-\beta_{2}^{2}\right)}{(\kappa+1) D(\dot{a})} \text { for } \quad i=1,2 \\
& \beta_{i}=\sqrt{1-\left(\frac{\dot{a}}{c_{i}}\right)^{2}} \text { and } D(\dot{a})=4 \beta_{1} \beta_{2}-\left(1+\beta_{2}^{2}\right)^{2},
\end{aligned}
$$

where $c_{1}$ and $c_{2}$ are the dilatational and shear wave speeds and $\kappa=3-4 \nu$ for plain strain.

By choosing the exact mode I or mode II asymptotic solution for $\left(\mathbf{u}^{\text {aux }}, \boldsymbol{\sigma}^{\text {aux }}\right)$, that is setting either $\left(K_{I}^{a u x}, K_{I I}^{a u x}\right)=(1,0)$ or $\left(K_{I}^{a u x}, K_{I I}^{a u x}\right)=(0,1)$, we can compute $K_{I}^{d y n}$ and $K_{I I}^{d y n}$.

For numerical implementation purposes, the interaction integral is calculated using a $J$-domain (see, for example, $[13,14]$ ). This $J$-domain is an external mesh centered at the crack tip and oriented by the local crack axis as shown in Figures A.1 and A.2.

\section{A.2 Fracture criteria}

From a simple point of view, crack growth simulation can be thought of as answering to three questions: when is the crack growing? how far? in which direction? 


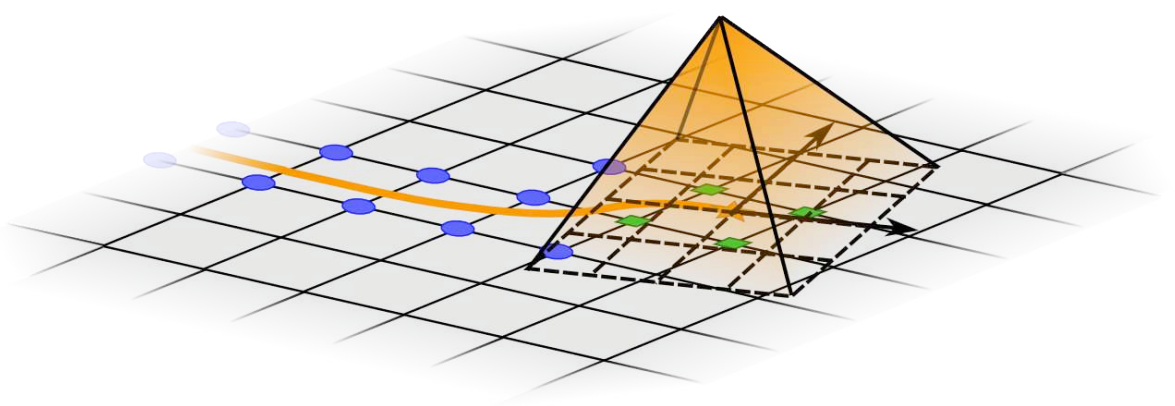

local coordinate system tangent to the crack lips

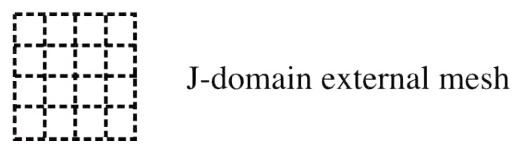

Fig. A.2. J-domain and virtual crack extension field used for interaction integral calculation.

As stated previously, we consider that brittle fracture is governed by dynamics stress intensity factors. A preferential direction $\theta^{*}$, defined as the direction of the maximum hoop stress (see, e.g., [23]), is assumed for the propagation of the crack. The analytical expression of this direction, in terms of dynamic SIFs is

$$
\theta^{*}=2 \arctan \left(\frac{1}{4}\left[\frac{K_{I}^{d y n}}{K_{I I}^{d y n}}-\operatorname{sign}\left(K_{I I}^{d y n}\right) \sqrt{8+\left(\frac{K_{I}^{d y n}}{K_{I I}^{d y n}}\right)^{2}}\right]\right) .
$$

Then, the intensity of the loading near the crack tip is calculated using the maximum hoop stress intensity factor $K^{*}$ corresponding to the hoop stress intensity factor in the preferential direction:

$$
K^{*}=\cos ^{3} \frac{\theta^{*}}{2}<K_{I}^{d y n}>-\frac{3}{2} \cos \frac{\theta^{*}}{2} K_{I I}^{d y n},
$$

where $\left\langle K_{I}^{d y n}>\right.$ is the positive part of $K_{I}^{d y n}$ and avoids any closure effects. Crack initiation occurs when this equivalent SIF reaches the dynamic initiation toughness $K_{I d}$. During the dynamic growth of a crack, the instantaneous maximum hoop stress intensity factor stays equal to the dynamic crack growth toughness $K_{1 D}$, which can now depend on the velocity $\dot{a}$ of the crack tip. Following Kanninen and Popelar [20] and Grégoire et al. [15], the dynamic crack growth toughness is defined as:

$$
K_{I D}(\dot{a})=\frac{K_{1 d}}{1-\frac{\dot{a}}{c_{r}}}
$$

where $c_{r}$, the Rayleigh wave speed, is the theoretical maximum velocity of a crack in a homogeneous medium. By setting $K^{*}$ equal to $K_{1 D}$, we obtain the 
following expression for the crack velocity:

$$
\dot{a}=c_{r}\left(1-\frac{K_{1 d}}{K^{*}}\right) .
$$

\section{References}

[1] I. Babuška and J.M. Melenk. The partition of unity method. International Journal for Numerical Methods in Engineering, 40:727-758, 1997.

[2] T. Belytschko and H. Chen. Singular enrichment finite element method for elastodynamic crack propagation. International Journal of Computational Methods, 1:1-15, 2004.

[3] T. Belytschko, H. Chen, J. Xu, and G. Zi. Dynamic crack propagation based on loss of hyperbolicity and a new discontinuous enrichment. International Journal for Numerical Methods in Engineering, 58:1873-1905, 2003.

[4] T. Black and T. Belytschko. Elastic crack growth in finite elements with minimal remeshing. International Journal for Numerical Methods in Engineering, 45:601-620, 1999.

[5] H.D. Bui. Mécanique de la rupture fragile. Masson, Paris, 1978.

[6] H.D. Bui, H. Maigre, and D. Rittel. A new approach to the experimental determination of the dynamic stress intensity factor. International Journal of Solids and Structures, 29(23):2881-2895, 1992.

[7] C. Daux, N. Moës, J. Dolbow, N. Sukumar, and T. Belytschko. Arbitrary branched and intersecting cracks with the extended finite element method. International Journal for Numerical Methods in Engineering, 48:1741-1760, 2000.

[8] R. de Borst, J.J.C. Remmers, and A. Needleman. Mesh independant discrete numerical representations of cohesive-zone models. Engineering fracture mechanics, 73(2):160-177, 2006.

[9] C.A. Duarte, O.N. Hamzeh, T.J. Liszka, and W.W. Tworzydlo. A generalized finite element method for the simulation of three-dimensional dynamic crack propagation. Computer Methods in Applied Mechanics and Engineering, 190:2227-2262, 2000.

[10] T. Elguedj, A. Gravouil, and A. Combescure. Appropriate extended functions for X-FEM simulation of plastic fracture mechanics. Computer Methods in Applied Mechanics and Engineering, 195:501-515, 2006.

[11] M. Fleming, Y.A. Chu, B. Moran, and T. Belytschko. Enriched elementfree galerkin methods for crack tip fields. International Journal for $\mathrm{Nu}$ merical Methods in Engineering, 40:1483-1504, 1997.

[12] L.B. Freund. Dynamic Fracture Mechanics. Cambridge University Press, Cambridge, UK, 1990.

[13] M. Gosz, J. Dolbow, and B. Moran. Domain integral formulation for stress intensity factor computation along curved three-dimensionnal interface 
cracks. International Journal of Solids and Structures, 35(15):1763-1783, 1998.

[14] A. Gravouil, N. Moës, and T. Belytschko. Non-planar 3D crack growth with the extended finite element and level sets - Part 2: Level set update. International Journal for Numerical Methods in Engineering, 54:25692586, 2002.

[15] D. Grégoire, H. Maigre, J. Réthoré, and A. Combescure. Dynamic crack propagation under mixed-mode loading - Comparison between experiments and X-FEM simulations. International Journal of Solids and Structures, 44:6517-6534, 2007.

[16] T.J.R. Hughes. The Finite Element Method: Linear Static and Dynamic Finite Element Analysis. Dover Publications, Mineola NY, 2000.

[17] T.J.R. Hughes and W.K. Liu. Implicit-explicit finite elements in transient analysis: implementation and numerical examples. Journal of Applied Mechanics, 45:375-378, 1978.

[18] G. Irwin. Analysis of stresses and strains near the end of a crack traversing a plate. Journal of Applied Mechanics, 24(3):361-364, 1957.

[19] J.F. Kalthoff. Modes of dynamic shear failure in solids. International Journal of Fracture, 101:1-31, 2000.

[20] M. Kanninen and C.H. Popelar. Advanced Fracture Mechanics. Oxford University Press, Oxford, UK, 1985.

[21] P. Krysl and T. Belytschko. The element free Galerkin method for dynamic propagation of arbitrary 3-D cracks. International Journal for Numerical Methods in Engineering, 44:767-800, 1999.

[22] Y.J. Lee and L.B. Freund. Fracture initiation due to asymmetric impact loading of an edge cracked plate. Journal of Applied Mechanics, 57:104111, 1990.

[23] H. Maigre and D. Rittel. Mixed-mode quantification for dynamic fracture initiation: application to the compact compression specimen. International Journal of Solids and Structures, 30(23):3233-3244, 1993.

[24] T. Menouillard, J. Réthoré, A. Combescure, and H. Bung. Efficient explicit time stepping for the extended finite element method. International Journal for Numerical Methods in Engineering, 68:911-938, 2006.

[25] T. Menouillard, J. Réthoré, N. Moës, A. Combescure, and H. Bung. Mass lumping strategies for X-FEM explicit dynamics: Application to crack propagation. International Journal for Numerical Methods in Engineering, 74(3):447-474, 2008.

[26] N. Moës, J. Dolbow, and T. Belytschko. A finite element method for crack growth without remeshing. International Journal for Numerical Methods in Engineering, 46:131-150, 1999.

[27] N. Moës, A. Gravouil, and T. Belytschko. Non-planar 3D crack growth with the extended finite element and level sets - Part 1: Mechanical model. International Journal for Numerical Methods in Engineering, 53:25492568, 2002.

[28] B. Moran and C.F. Shih. Crack tip and associated domain integral from 
momentum and energy balance. Engineering Fracture Mechanics, 27:615642, 1987.

[29] N. Newmark. A method of computation for structural dynamics. Journal of the Engineering Mechanics Division, Proceedings of the ASCE, 85(EM3):67-94, 1959.

[30] B. Prabel, A. Combescure, A. Gravouil, and S. Marie. Level set XFEM non matching meshes: Application to dynamic crack propagation in elastic-plastic media. International Journal for Numerical Methods in Engineering, 69:1553-1569, 2007.

[31] B.N. Rao and S. Rahman. An enriched meshless method for non-linear fracture mechanics. International Journal for Numerical Methods in Engineering, 50:197-223, 2004.

[32] J.J.C. Remmers, R. de Borst, and A. Needleman. The simulation of dynamic crack propagation using the cohesive segments method. Journal of the Mechanics and Physics of Solids, 56:70-92, 2008.

[33] J. Réthoré, A. Gravouil, and A. Combescure. A combined space-time extended finite element method. International Journal for Numerical Methods in Engineering, 64:260-284, 2005.

[34] J. Réthoré, A. Gravouil, and A. Combescure. An energy-conserving scheme for dynamic crack growth using the extended finite element method. International Journal for Numerical Methods in Engineering, 63:631-659, 2005.

[35] P. Rozycki, N. Moës, E. Béchet, and C. Dubois. X-FEM explicit dynamics for contant strain elements to alleviate mesh constraints on internal or external boundaries. Computer Methods in Applied Mechanics and Engineering, 197:349-363, 2008.

[36] T.H. Seelig, D. Gross, and K. Pothmann. Numerical simulation of a mixed-mode dynamics fracture experiment. International Journal of Fracture, 99:325-338, 1999.

[37] N. Sukumar, D. Chopp, N. Moës, and T. Belytschko. Modeling holes and inclusions by level sets in the extended finite element method. Computer Methods in Applied Mechanics and Engineering, 190:6183-6200, 2000.

[38] N. Sukumar, N. Moës, B. Moran, and T. Belytschko. Extended finite element method for three-dimensional crack modeling. International Journal for Numerical Methods in Engineering, 48:1549-1570, 2000.

[39] G. Zi, H. Chen, J. Xu, and T. Belytschko. The extended finite element method for dynamic fractures. Shock and Vibration, 12:9-23, 2005. 